Available online at www.sciencedirect.com

science (d) Directo

\title{
Advances in wide bandgap materials for semiconductor spintronics
}

\author{
S.J. Pearton ${ }^{1, *}$, C.R. Abernathy ${ }^{1}$, D.P. Norton $^{1}$, A.F. Hebard ${ }^{2}$, \\ Y.D. Park ${ }^{3}$, L.A. Boatner ${ }^{4}$, J.D. Budai ${ }^{4}$ \\ ${ }^{1}$ Department of Materials Science and Engineering, University of Florida, P.O. Box 116400, \\ 100 Rhines Hall, Gainesville, FL 32611-6400, USA \\ ${ }^{2}$ Department of Physics, University of Florida, Gainesville, FL 32611, USA \\ ${ }^{3}$ Center for Strongly Correlated Materials Research, Seoul National University, Seoul 151-747, South Korea \\ ${ }^{4}$ Oak Ridge National Laboratory, Oak Ridge, TN 37813, USA
}

\begin{abstract}
Existing semiconductor electronic and photonic devices utilize the charge on electrons and holes in order to perform their specific functionality such as signal processing or light emission. The relatively new field of semiconductor spintronics seeks, in addition, to exploit the spin of charge carriers in new generations of transistors, lasers and integrated magnetic sensors. The ability to control of spin injection, transport and detection leads to the potential for new classes of ultra-low power, high speed memory, logic and photonic devices. The utility of such devices depends on the availability of materials with practical $(>300 \mathrm{~K})$ magnetic ordering temperatures. In this paper, we summarize recent progress in dilute magnetic semiconductors (DMS) such as (Ga, Mn)N, (Ga, Mn)P, (Zn, $\mathrm{Mn}) \mathrm{O}$ and $(\mathrm{Zn}, \mathrm{Mn}) \mathrm{SiGeN}_{2}$ exhibiting room temperature ferromagnetism, the origins of the magnetism and its potential applications in novel devices such as spin-polarized light emitters and spin field effect transistors.

(C) 2003 Elsevier Science B.V. All rights reserved.
\end{abstract}

Keywords: Wide bandgap materials; Semiconductor; Spintronics

\section{Introduction-what is spintronics?}

Two of the most successful technologies in existence today have created the Si integrated circuit (ICs) industry and the data storage industry. Both continue to advance at a rapid pace. In the case of ICs, the number of transistors on a chip doubles about every 18 months according to Moore's law. For magnetic hard disk drive technology, a typical desk-top computer drive today has a 40GB per disk capacity, whereas in 1995 this capacity was $\sim 1 \mathrm{~GB}$ per disk. Since 1991, the overall bit density on a magnetic head has increased at an annual rate of $60-100 \%$ and is currently $\sim 10.7$ Gbits/in. ${ }^{2}$ (see, for example [1]). The integrated circuits operate by controlling the flow of carriers through the semiconductor by applied electric fields. The key parameter therefore is the charge on the electrons or holes. For the case of magnetic data storage, the key parameter is the spin of the electron, as spin can be thought of as the fundamental origin of magnetic moment. The characteristics of ICs include high speed signal processing and excellent reliability, but the memory elements are volatile (the stored information is lost when the power is switched-off, as data is stored as charge in capacitors, i.e. DRAMs). A key advantage of magnetic memory technologies is that they are non-volatile since they employ ferromagnetic materials which by nature have remanence.

\footnotetext{
${ }^{*}$ Corresponding author. Tel.: +1-352-846-1086; fax: +1-352-846-1660.

E-mail address: spear@mse.ufl.edu (S.J. Pearton).
} 


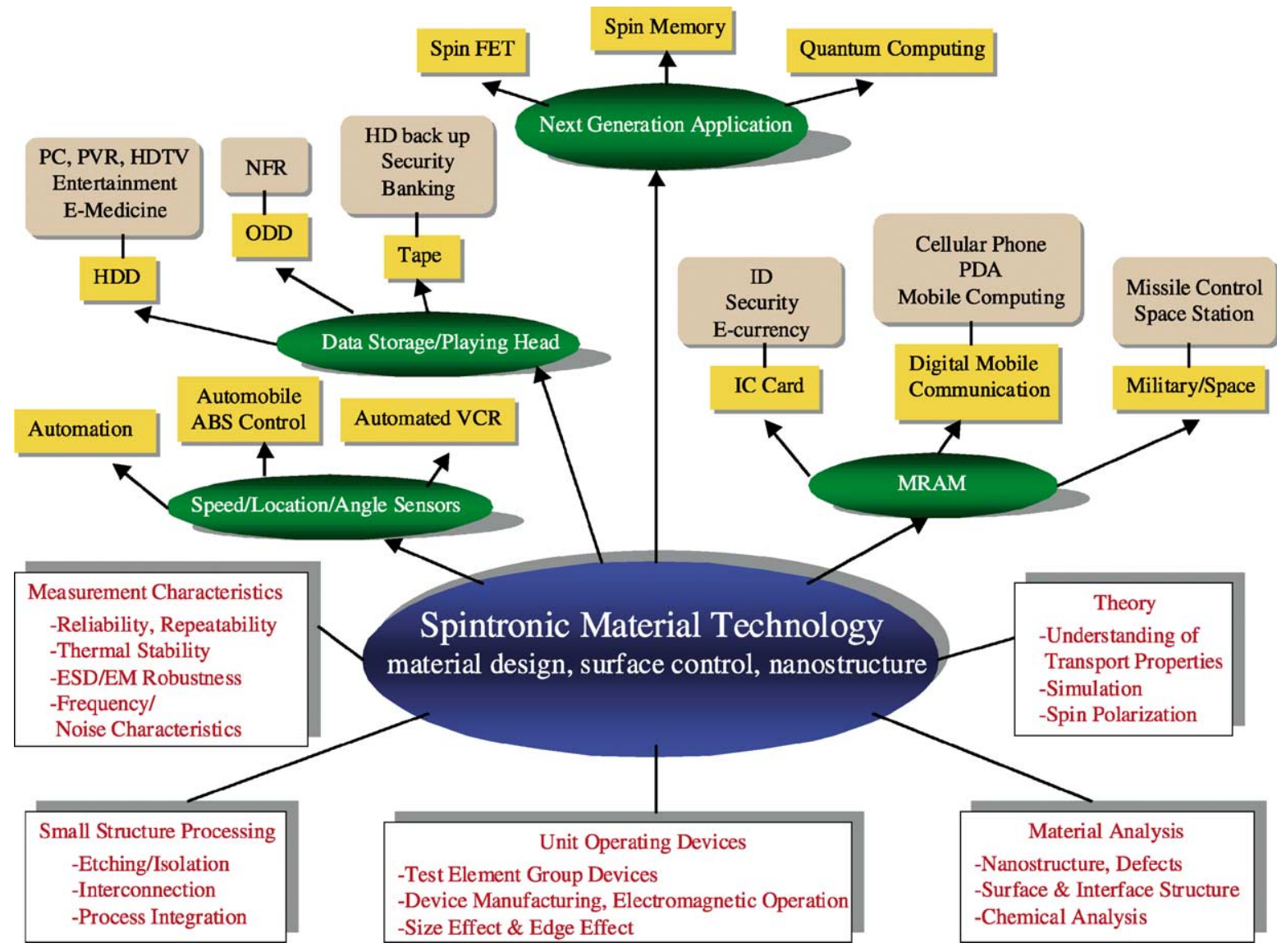

Fig. 1. Technology tree for spin-based devices and their potential applications (after http://spintronics.korea.ac.kr/ research_map1.htm).

The emerging field of semiconductor spin transfer electronics (spintronics) seeks to exploit the spin of charge carriers in semiconductors. It is widely expected that new functionalities for electronics and photonics can be derived if the injection, transfer and detection of carrier spin can be controlled above room temperature. Among this new class of devices are spin transistors operating at very low powers for mobile applications that rely on batteries, optical emitters with encoded information through their polarized light output, fast non-volatile semiconductor memory and integrated magnetic/electronic/photonic devices ("electromagnetism-on-a-chip"). A proposed technology tree for spin-based devices is shown in Fig. 1. Since the magnetic properties of ferromagnetic semiconductors are a function of carrier concentration in the material in many cases, then it will be possible to have electrically or optically-controlled magnetism through field-gating of transistor structures or optical excitation to alter the carrier density. This novel control of magnetism has already been achieved electronically and optically in an InMnAs metal-insulator semiconductor structure at low temperatures [2,3] and electronically in Mn:Ge [4]. A number of recent reviews have covered the topics of spin injection, coherence length and magnetic properties of materials systems such as $(\mathrm{Ga}, \mathrm{Mn}) \mathrm{As}$ [5-7], ( $\mathrm{In}, \mathrm{Mn}) \mathrm{As}$ [5-7] and $(\mathrm{Co}, \mathrm{Ti}) \mathrm{O}_{2}$ [8] and the general areas of spin injection from metals into semiconductors and applications of the spintronic phenomena [9-12]. The current interest in magnetic semiconductors can be traced to difficulties in injecting spins from a ferromagnetic metal into a semiconductor $[13,14]$, which idea can be traced to fruitful research in 
epitaxial preparation of ferromagnetic transitional metals on semiconductor substrates [15]. A theory first proposed by Schmidt et al. [16] points out that due to the dissimilar materials properties of a metal and semiconductor, an efficient spin injection in the diffusive transport regime is difficult unless the magnetic material is nearly $100 \%$ spin-polarized, i.e. half-metallic [17]. Although there have been recent reports of successful and efficient spin injection from a metal to a semiconductor even at room temperature by ballistic transport (i.e. Schottky barriers and tunneling) [18], the realization of functional spintronic devices requires materials with ferromagnetic ordering at operational temperatures compatible with existing semiconductor materials.

\section{Materials selection}

There are two major criteria for selecting the most promising materials for semiconductor spintronics. First, the ferromagnetism should be retained to practical temperatures (i.e. $>300 \mathrm{~K}$ ). Second, it would be a major advantage if there were already an existing technology base for the material in other applications. Most of the work in the past has focused on (Ga, Mn)As and (In, Mn)As. There are indeed major markets for their host materials in infra-red light-emitting diodes and lasers and high speed digital electronics (GaAs) and magnetic sensors (InAs). Most of the past attention on ferromagnetic semiconductors focussed on the (Ga, Mn)As [19-42] and (In, Mn)As [43-50] systems. In samples carefully grown single-phase by molecular beam epitaxy (MBE), the highest Curie temperatures reported are $\sim 110 \mathrm{~K}$ for $(\mathrm{Ga}, \mathrm{Mn}) \mathrm{As}$ and $\sim 35 \mathrm{~K}$ for (In, Mn)As. For ternary alloys such as $\left(\mathrm{In}_{0.5} \mathrm{Ga}_{0.5}\right)_{0.93} \mathrm{Mn}_{0.07} \mathrm{As}$, the Curie temperature is also low $\sim 110 \mathrm{~K}$ [51]. A tremendous amount of research on these materials systems has led to some surprising results, such as the very long spin lifetimes and coherence times in GaAs [4] and the ability to achieve spin transfer through a heterointerface [52-69], either of semiconductor-semiconductor or metal-semiconductor. One of the most effective methods for investigating spin-polarized transport is by monitoring the polarized electroluminescence output from a quantum well light-emitting diode into which the spin current is injected. Quantum selection rules relating the initial carrier spin polarization and the subsequent polarized optical output can provide a quantitative measure of the injection efficiency $[67,69,70]$.

There are a number of essential requirements for achieving practical spintronic devices in addition to the efficient electrical injection of spin-polarized carriers. These include the ability to transport the carriers with high transmission efficiency within the host semiconductor or conducting oxide, the ability to detect or collect the spin-polarized carriers and to be able to control the transport through external means such as biasing of a gate contact on a transistor structure. The observation of spin current-induced switching in magnetic heterostructures is an important step in realizing practical devices [71]. Similarly, Nitta et al. [72] demonstrated that a spin-orbit interaction in a semiconductor quantum well could be controlled by applying a gate voltage. These key aspects of spin injection, spin-dependent transport, manipulation and detection form the basis of current research and future technology. The use of read sensors based on metallic spin valves in disk drives for magnetic recording is already a US\$ 100 billion per year industry. It should also be pointed out that spintronic effects are inherently tied to nanotechnology, because of the short $(\sim 1 \mathrm{~nm})$ characteristic length of some of the magnetic interactions. Combined with the expected low power capability of spintronic devices, this should lead to extremely high packing densities for memory elements. A recent review of electronic spin injection, spin transport and spin detection technologies has recently been given by Buhrman and co-workers [6], as part of a very detailed and comprehensive study of the status and trends of research into spin electronics in Japan, Europe and 
the US. The technical issues covered fabrication and characterization of magnetic nanostructures, magnetism and spin control in these structures, magneto-optical properties of semiconductors and magneto-electronics and devices. The non-technical issues covered included industry and academic cooperation and long-term research challenges. The panel findings are posted on the web site [7].

In this review, we focus on a particular and emerging aspect of spintronics, namely recent developments in achieving practical magnetic ordering temperatures in technologically useful semiconductors [73-79]. While the progress in synthesizing and controlling the magnetic properties of III-arsenide semiconductors has been astounding, the reported Curie temperatures are too low to have significant practical impact. A key development that focused attention on wide bandgap semiconductors as being the most promising for achieving high Curie temperatures was the work of Dietl et al. [80]. They employed the original Zener model of ferromagnetism [81] to predict $T_{\mathrm{C}}$ values exceeding room temperature for materials such as $\mathrm{GaN}$ and $\mathrm{ZnO}$ containing $5 \%$ of $\mathrm{Mn}$ and a high hole concentration $\left(3.5 \times 10^{20} \mathrm{~cm}^{-3}\right)$.

Other materials for which room temperature ferromagnetism has been reported include $(\mathrm{Cd}$, $\mathrm{Mn}) \mathrm{GeP}_{2}$ [74], ( $\left.\mathrm{Zn}, \mathrm{Mn}\right) \mathrm{GeP}_{2}$ [75], $\mathrm{ZnSnAs}_{2}$ [76], ( $\left.\mathrm{Zn}, \mathrm{Co}\right) \mathrm{O}$ [77] and (Co, Ti) $\mathrm{O}_{2}$ [8,78] as well as Eu chalcogenides and others that have been studied in the past [79]. Some of these chalcopyrites and wide bandgap oxides have interesting optical properties, but they lack a technology and experience base as large as that of most semiconductors.

The key breakthrough that focused attention on wide bandgap semiconductors as being the most promising for achieving practical ordering temperatures was the theoretical work of Dietl et al. [80]. They predicted that cubic GaN doped with $\sim 5$ at.\% of $\mathrm{Mn}$ and containing a high concentration of holes $\left(3.5 \times 10^{20} \mathrm{~cm}^{-3}\right)$ should exhibit a Curie temperature exceeding room temperature. In the period following the appearance of this work, there has been tremendous progress on both the realization of high-quality $(\mathrm{Ga}, \mathrm{Mn}) \mathrm{N}$ epitaxial layers and on the theory of ferromagnetism in these so-called dilute magnetic semiconductors (DMS). The term DMS refers to the fact that some fraction of the atoms in a non-magnetic semiconductor like GaN are replaced by magnetic ions. A key, unanswered question is whether the resulting material is indeed an alloy of $(\mathrm{Ga}, \mathrm{Mn}) \mathrm{N}$ or whether it remains as $\mathrm{GaN}$ with clusters, precipitates or second phases that are responsible for the observed magnetic properties [82].

\section{Mechanisms of ferromagnetism}

Fig. 2 shows some of the operative mechanisms for magnetic ordering in DMS materials. Two basic approaches to understanding the magnetic properties of dilute magnetic semiconductors have emerged. The first class of approaches is based on mean-field theory which originates in the original model of Zener [81]. The theories that fall into this general model implicitly assume that the dilute magnetic semiconductor is a more-or-less random alloy, e.g. $(\mathrm{Ga}, \mathrm{Mn}) \mathrm{N}$, in which $\mathrm{Mn}$ substitutes for one of the lattice constituents. Within these theories, there are differences in how the free carriers are assumed to interact, as shown in Fig. 3. The second class of approaches suggests that the magnetic atoms form small (a few atoms) clusters that produce the observed ferromagnetism [82]. A difficulty in experimentally verifying the mechanism responsible for the observed magnetic properties is that depending on the growth conditions employed for growing the DMS material, it is likely that one could readily produce samples that span the entire spectrum of possibilities from single-phase random alloys to nanoclusters of the magnetic atoms to precipitates and second phase formation. Therefore, it is necessary to decide on a case-by-case basis which mechanism is applicable. This can only be achieved by a careful correlation of the measured magnetic properties with materials 
(A)
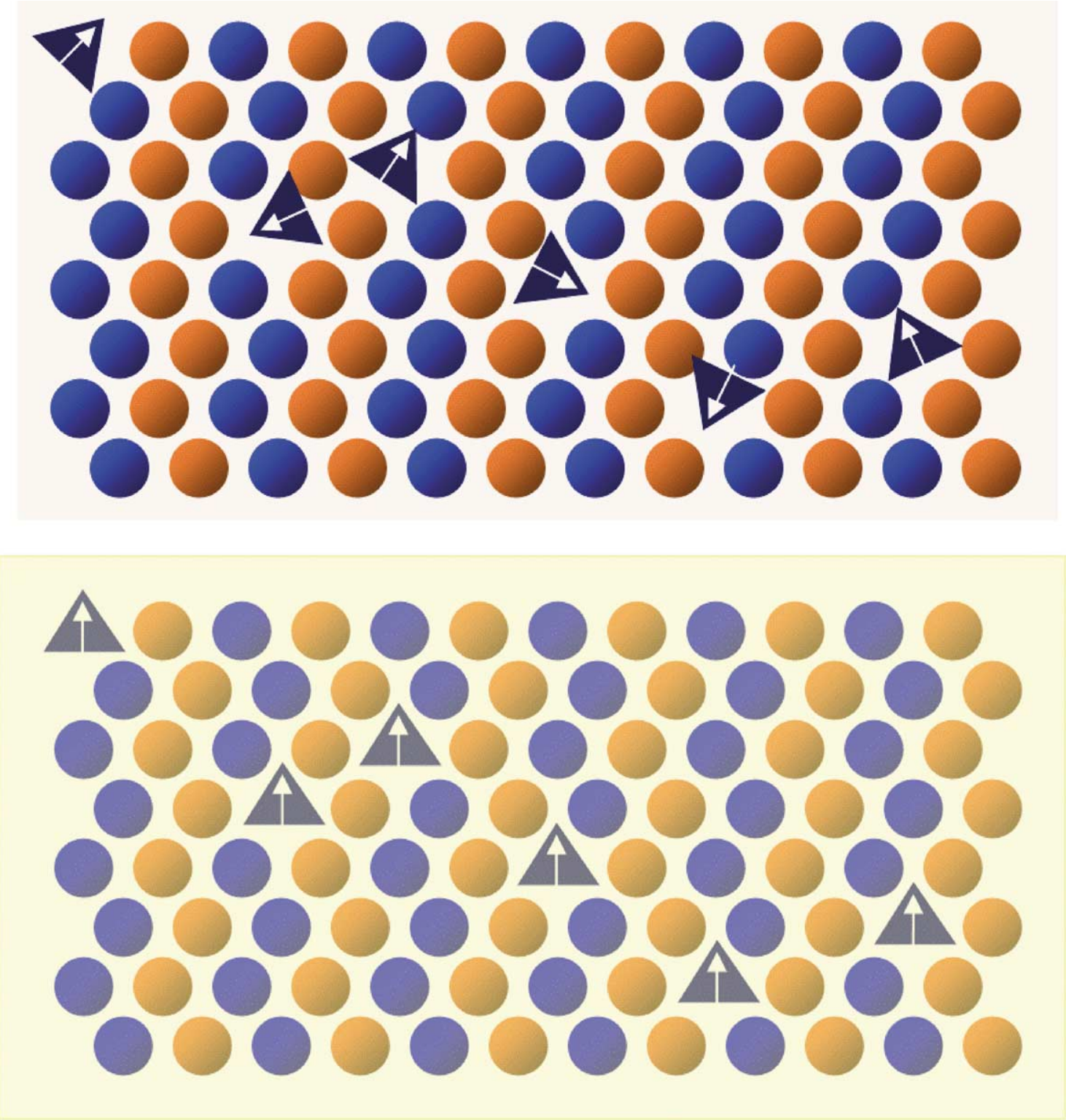

(B)

Fig. 2. Semiconductor matrix with high concentrations of magnetic impurities (i.e. Mn), randomly distributed (defects), can be insulators (A) for group II-VI materials where divalent Mn ions occupy group II sites. At high concentrations, Mn ions are antiferromagnetically coupled, but at dilute limits, atomic distances between magnetic ions are large, and antiferromagnetic coupling is weak. For the cases where there is high concentrations of carriers (B) (i.e. (Ga, Mn)As where Mn ions behave as acceptors and provide magnetic moment as they occupy trivalent Ga sites), the carriers are thought to mediate ferromagnetic coupling between magnetic ions. Between near insulating and metallic cases, at low carrier regimes, hole carrier concentrations are localized near the magnetic impurity. Below certain temperatures, a percolation network (C) is formed in which clusters the holes are delocalized and hop from site to site, which energetically favors maintaining the carriers' spin orientation during the process, an effective mechanism for aligning Mn moments within the cluster network. Alternatively, at percolation limits, localized hole near the magnetic impurity is polarized, and the energy of the system is lowered when the polarization of the localized holes are parallel (D). 
(C)

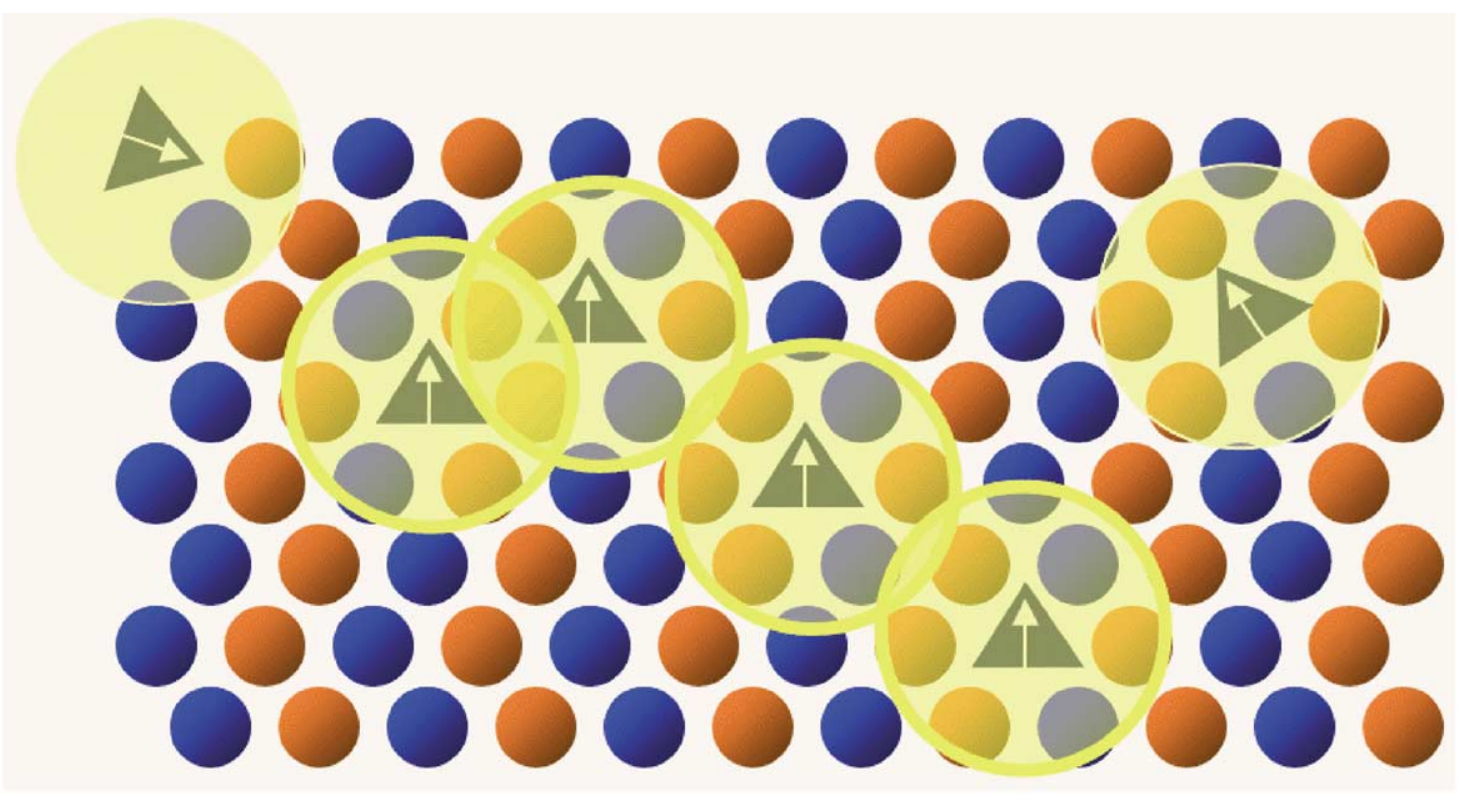

(D)

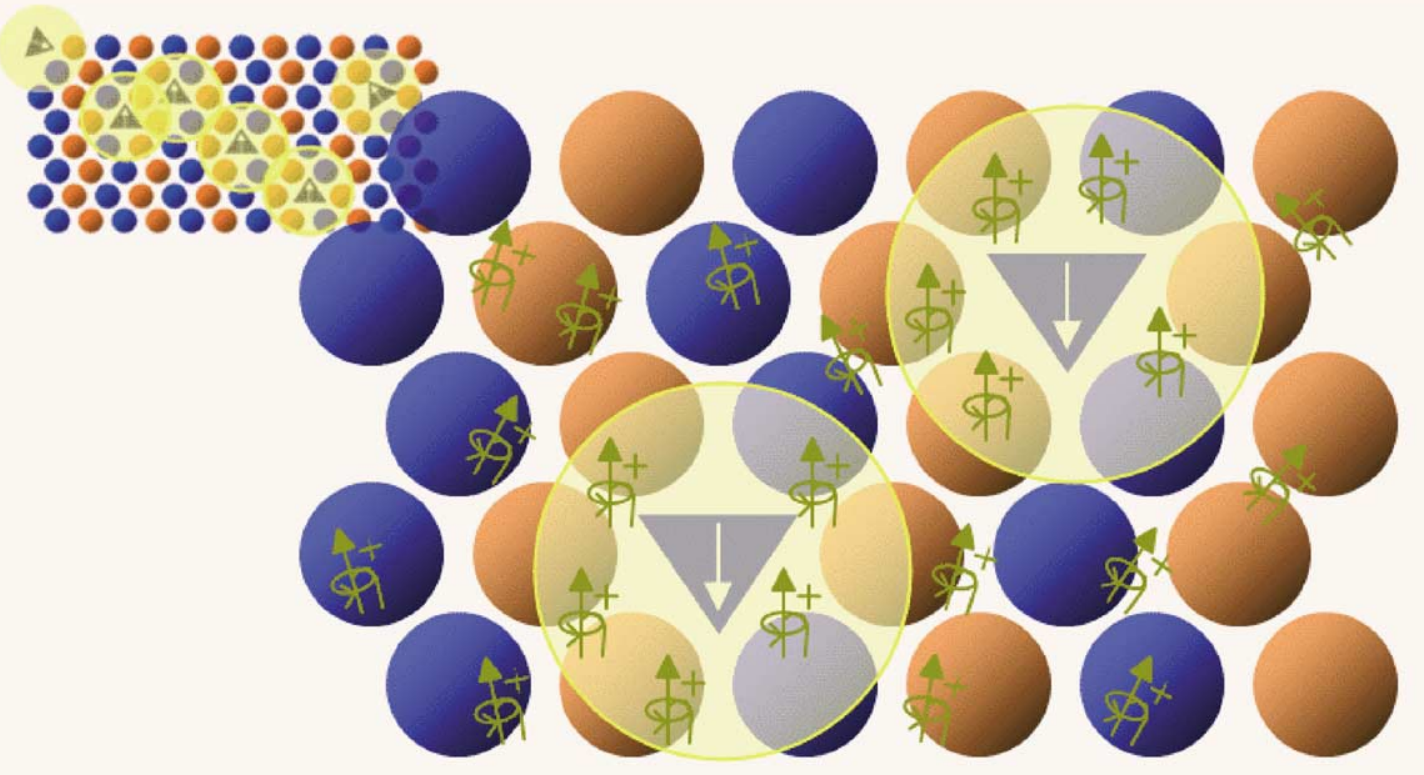

Fig. 2. (Continued).

analysis methods that are capable of detecting other phases or precipitates. If, for example, the magnetic behavior of the DMS is characteristic of that of a known ferromagnetic second phase (such as $\mathrm{MnGa}$ or $\mathrm{Mn}_{4} \mathrm{~N}$ in $\left.(\mathrm{Ga}, \mathrm{Mn}) \mathrm{N}\right)$, then clearly the mean-field models are not applicable. To date, most experimental reports concerning room temperature ferromagnetism in DMS employ X-ray diffraction, selected-area diffraction patterns (SADP), transmission electron microscopy (TEM), photoemission or X-ray absorption (including extended X-ray absorption fine structure (EXAFS) as discussed later) to determine whether the magnetic atoms are substituting for one of the lattice constituents to form an alloy. Given the level of dilution of the magnetic atoms, it is often very 


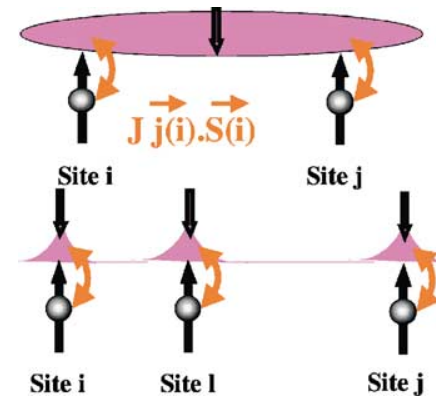

Scenario 1 : Delocalized holes form a Ferm liquid. Coulomb effects are approximated. Effective medium theory.

(Dietl, Konig/Schliemann/MacDonald, ...)

Scenario 2 : Holes form an almost localized tight-binding band. Fluctuation effects due to randomness and hole-hole correlations are approximated.

(Berciu/Bhatt, Kaminski/Das Sarma, Dagotto, ...

Fig. 3. Schematic of role of carriers (holes) in the various theories for carrier-induced ferromagnetism in dilute magnetic III-V semiconductors.

difficult to categorically determine the origin of the ferromagnetism. Indirect means such as superconducting quantum interference device (SQUID) magnetometer measurements to exclude any ferromagnetic intermetallic compounds as the source of magnetic signals and even the presence of what is called the anomalous or extraordinary Hall effect, that have been widely used to verify a single-phase system, may be by itself insufficient to characterize a DMS material. It could also certainly be the case that magnetically-active clusters or second phases could be present in a pseudorandom alloy and therefore that several different mechanisms could contribute to the observed magnetic behavior. There is a major opportunity for the application of new, element- and lattice position-specific analysis techniques, such as the various scanning tunneling microscopies and Zcontrast scanning transmission electron microscopy (Z-contrast STEM) amongst others for revealing a deeper microscopic understanding of this origin of ferromagnetism in the new DMS materials.

The mean-field approach basically assumes that the ferromagnetism occurs through interactions between the local moments of the Mn atoms, which are mediated by free holes in the material. The spin-spin coupling is also assumed to be a long-range interaction, allowing use of a mean-field approximation $[80,83,84]$. In its basic form, this model employs a virtual-crystal approximation to calculate the effective spin-density due to the $\mathrm{Mn}$ ion distribution. The direct $\mathrm{Mn}-\mathrm{Mn}$ interactions are antiferromagnetic so that the Curie temperature, $T_{\mathrm{C}}$, for a given material with a specific $\mathrm{Mn}$ concentration and hole density (derived from Mn acceptors and/or intentional shallow level acceptor doping), is determined by a competition between the ferromagnetic and antiferromagnetic interactions. In the presence of carriers, $T_{\mathrm{C}}$ is given by the expression $[80,85]$

$$
T_{\mathrm{C}}=\left\lfloor\frac{\mathrm{N}_{\mathrm{O}} \operatorname{Xeff} S(S+1) \beta^{2} A_{\mathrm{F}} P_{\mathrm{S}}\left(T_{\mathrm{C}}\right)}{12 k_{\mathrm{B}}}\right\rfloor-T_{\mathrm{AF}}
$$

where $\mathrm{N}_{\mathrm{O}}$ Xeff is the effective spin concentration, $S$ the localized spin state, $\beta$ the $\mathrm{p}-\mathrm{d}$ exchange integral, $A_{\mathrm{F}}$ the Fermi liquid parameter, $P_{\mathrm{S}}$ the total density of states, $k_{\mathrm{B}}$ is Boltzmann's constant and $T_{\mathrm{AF}}$ describes the contribution of antiferromagnetic interactions. Numerous refinements of this approach have appeared recently, taking into account the effects of positional disorder [86,87], indirect exchange interactions [88], spatial inhomogeneities and free-carrier spin polarization [89,90]. Fig. 4 shows a compilation of the predicted $T_{\mathrm{C}}$ values, together with some reported experimental values. In the subsequent period after appearance of the Dietl et al. [80] paper, remarkable progress has been made on the realization of materials with $T_{\mathrm{C}}$ values at or above room temperature.

The mean-field model and its variants produces reliable estimates of $T_{\mathrm{C}}$ for materials such as $(\mathrm{Ga}, \mathrm{Mn}) \mathrm{As}$ and $(\mathrm{In}, \mathrm{Mn}) \mathrm{As}$ and predicts that $(\mathrm{Ga}, \mathrm{Mn}) \mathrm{N}$ will have a value above room temperature 


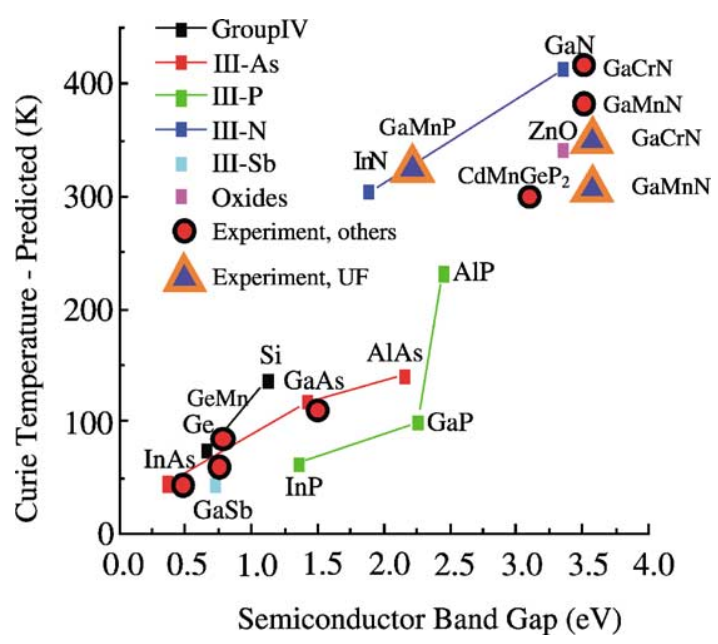

Fig. 4. Predicted Curie temperatures as a function of bandgap (after [80]), along with some experimentally reported values in the literature.

[80]. Examples of the predicted ferromagnetic transition temperatures for both $(\mathrm{Ga}, \mathrm{Mn}) \mathrm{As}$ and $(\mathrm{Ga}$, $\mathrm{Mn}) \mathrm{N}$ are shown in Fig. 5 for four different variants of the mean-field approach [91]. These are the standard mean-field theory $\left(T_{\mathrm{C}}^{\mathrm{MF}}\right)$, a version that accounts for the role of Coulomb interactions with holes in the valence band (exchange-enhanced, $T_{\mathrm{C}}^{\mathrm{X}}$ ), another version that accounts for correlations in $\mathrm{Mn}$ ion orientations (collective, $T_{\mathrm{C}}^{\mathrm{coll}}$ ) or an estimate based on where excited spin waves cancel out the total spin of the ground state $\left(T_{\mathrm{C}}^{\text {est }}\right)$ [91]. Note that the dependence of any of the calculated $T_{\mathrm{C}}$ values on hole density in the material is much steeper for $(\mathrm{Ga}, \mathrm{Mn}) \mathrm{As}$ than for $(\mathrm{Ga}, \mathrm{Mn}) \mathrm{N}$. The range of predicted values for GaAs has a much higher distribution than for GaN. This data emphasizes the point that the mean-field theories produce fairly reliable predictions for $(\mathrm{Ga}, \mathrm{Mn}) \mathrm{As}$, but at this stage are not very accurate for $(\mathrm{Ga}, \mathrm{Mn}) \mathrm{N}$.

A second point largely overlooked in the theoretical work to date is that fact that the assumed hole densities may not be realistic. While GaAs can be readily doped with shallow acceptors such as $\mathrm{C}$ to produce hole densities of around $10^{21} \mathrm{~cm}^{-3}$ [92] and the Mn acceptors also contribute holes, the p-doping levels in $\mathrm{GaN}$ are limited to much lower values under normal conditions. For example, the ionization level $\left(E_{\mathrm{a}}\right)$ of the most common acceptor dopant in $\mathrm{GaN}$, namely $\mathrm{Mg}$, is relatively deep in the gap $\left(E_{\mathrm{v}}+0.17 \mathrm{eV}\right)$. Since the number of holes $(P)$ is determined by the fraction of acceptors that are actually ionized at a given temperature $T$ through a Boltzmann factor

$$
P \propto \exp \left(-\frac{E_{\mathrm{a}}}{k T}\right)
$$

then for $\mathrm{Mg}$ at room temperature only a few percent of acceptors are ionized. While the $\mathrm{Mg}$ acceptor concentration in $\mathrm{GaN}$ can exceed $10^{19} \mathrm{~cm}^{-3}$, a typical hole concentration at $25^{\circ} \mathrm{C}$ is $P \sim 3 \times$ $10^{17} \mathrm{~cm}^{-3}$. Initial reports of the energy level of $\mathrm{Mn}$ in $\mathrm{GaN}$ show it is very deep in the gap, $E_{\mathrm{v}}+1.4 \mathrm{eV}$ [93], and thus would be an ineffective dopant under most conditions. Some strategies for enhancing the hole concentration do exist, such as co-doping both acceptors and donors to reduce self-compensation effects [94] or the use of selectively-doped $\mathrm{AlGaN} / \mathrm{GaN}$ superlattices in which there is transfer of free holes from $\mathrm{Mg}$ acceptors in the AlGaN barriers to the GaN quantum wells [95]. These methods appear capable under optimum conditions of increasing the hole density in GaN to $>10^{18} \mathrm{~cm}^{-3}$ at $25^{\circ} \mathrm{C}$. 

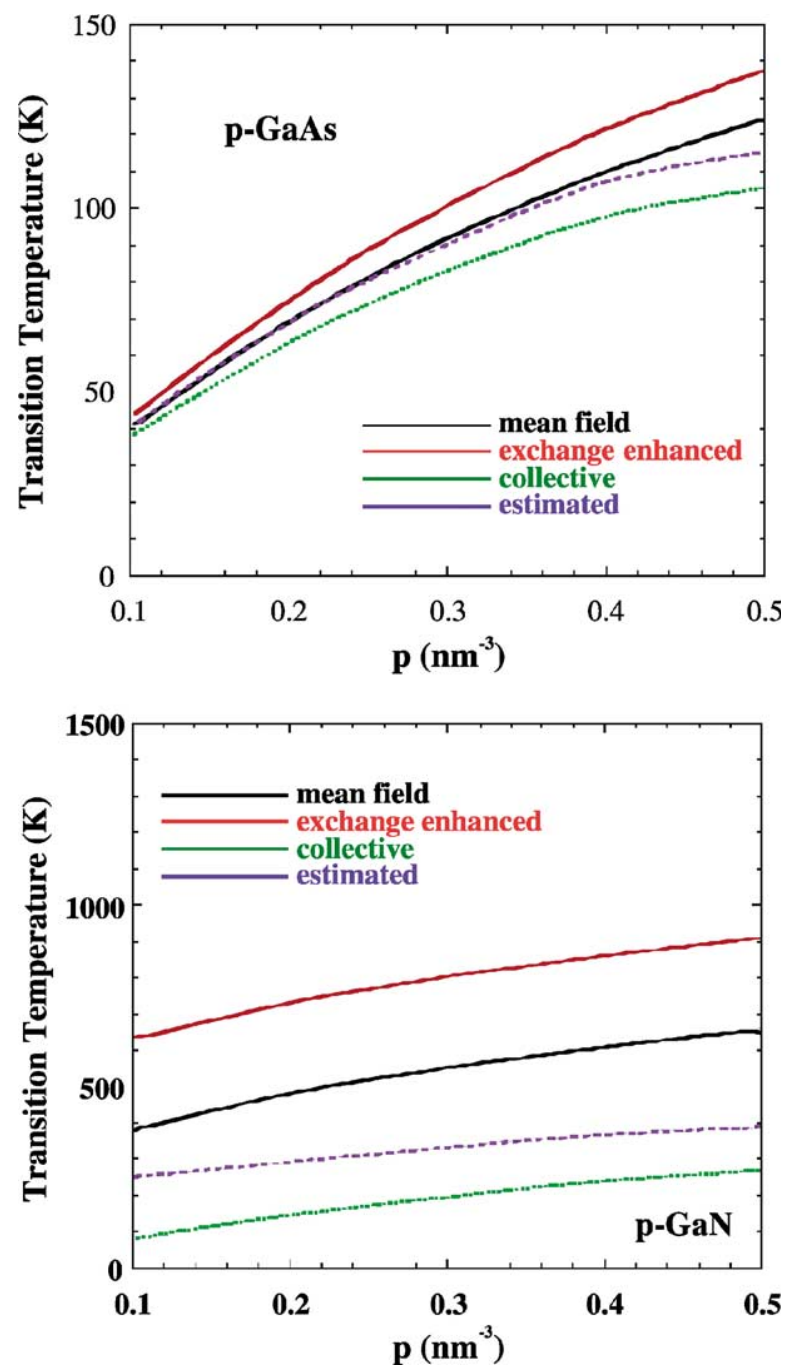

Fig. 5. Predicted ferromagnetic transition temperatures in (Ga, Mn)As (top) or (Ga, Mn)N (bottom) containing 5 at.\% Mn, as a function of hole density. The four different curves in each graph represent results obtained from different variants of mean-field theory (after [91]).

A further issue that needs additional exploration in the theories is the role of electrons, rather than holes, in stabilizing the ferromagnetism in DMS materials. All of the reports of ferromagnetism in $(\mathrm{Ga}, \mathrm{Mn}) \mathrm{N}$, for example, occur for material that is actually n-type. Since the material has to be grown at relatively low temperatures to avoid Mn precipitation and therefore only molecular beam epitaxy (MBE) can be used, there is always the possibility of unintentional n-type doping from nitrogen vacancies, residual lattice defects or impurities such as oxygen. Therefore, stoichiometry effects, crystal defects or unintentional impurities may control the final conductivity, rather than $\mathrm{Mn}$ or the intentionally-introduced acceptor dopants. Once again, this is much less of an issue in materials such as GaAs, whose low temperature growth is relatively well understood and controlled.

While most of the theoretical work for DMS materials has focused on the use of Mn as the magnetic dopant, there has been some progress on identifying other transition metal (TM) atoms that 


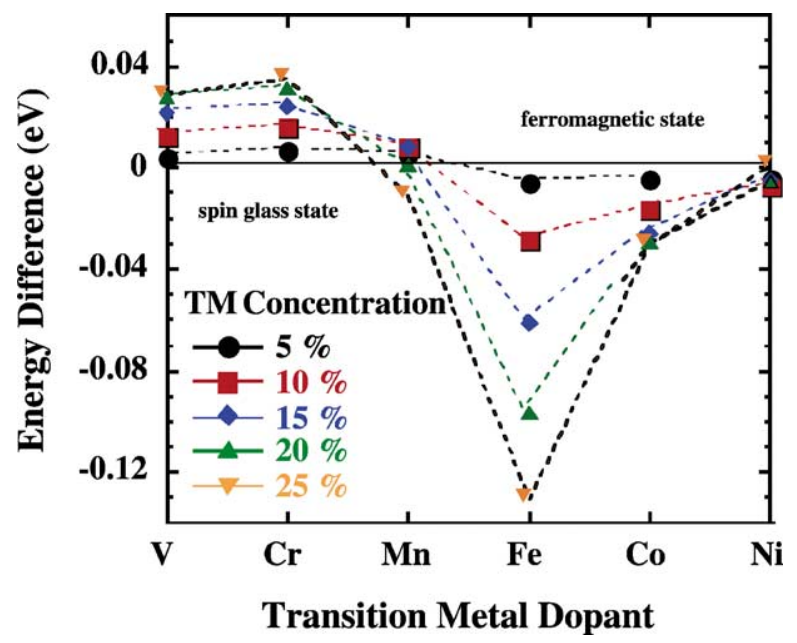

Fig. 6. Predicted stability of the ferromagnetic states of different transition metal (TM) atoms in GaN as a function of transition metal concentration. The vertical axis represents the energy difference between the ferromagnetic and spin-glass states for each metal atom (after [96]).

may be effective. Fig. 6 shows the predicted stability of ferromagnetic states in GaN doped with different $3 \mathrm{~d}$ transition metal atoms [96]. The results are based on a local spin-density approximation which assumed that $\mathrm{Ga}$ atoms were randomly substituted with the magnetic atoms and did not take into account any additional carrier doping effects. In this study it was found that $(\mathrm{Ga}, \mathrm{V}) \mathrm{N}$ and $(\mathrm{Ga}$, $\mathrm{Cr}) \mathrm{N}$ showed stable ferromagnetism for all transition metal concentrations whereas $\mathrm{Fe}$, $\mathrm{Co}$ or $\mathrm{Ni}$ doping produced spin-glass ground states [96]. For the case of $\mathrm{Mn}$, the ferromagnetic state was the lowest energy state for concentrations up to $\sim 20 \%$, whereas the spin-glass state became the most stable at higher Mn concentrations.

\section{1. $(G a, M n) P$}

Ferromagnetism above room temperature in $(\mathrm{Ga}, \mathrm{Mn}) \mathrm{P}$ has been reported for two different methods of Mn incorporation, namely ion implantation [97] and doping during MBE growth [97,98] The implantation process is an efficient one for rapidly screening whether particular combinations of magnetic dopants and host semiconductors are promising in terms of ferromagnetic properties. We have used implantation to introduce ions such as $\mathrm{Mn}, \mathrm{Fe}$ and $\mathrm{Ni}$ into a variety of substrates, including $\mathrm{GaN}, \mathrm{SiC}$ and $\mathrm{GaP}$.

The temperature-dependent magnetization of a strongly $\mathrm{p}$-type $\left(\mathrm{p} \sim 10^{20}\right)$, carbon-doped $\mathrm{GaP}$ sample implanted with $\sim 6$ at. $\%$ of $\mathrm{Mn}$ and then annealed at $700{ }^{\circ} \mathrm{C}$, is shown in Fig. 7 . The diamagnetic contribution was subtracted from the background. A Curie temperature $\left(T_{\mathrm{C}}\right)$ of $\sim 270 \mathrm{~K}$ is indicated by the dashed vertical line, while the inset shows a ferromagnetic Curie temperature of $236 \mathrm{~K}$.

Examples of hysteresis loops from MBE-grown samples doped during growth are shown in Fig. 8. The hysteresis could be detected to $330 \mathrm{~K}$. No secondary phases (such as $\mathrm{MnGa}$ or MnP) or clusters were determined by transmission electron microscopy, X-ray diffraction or selected-area diffraction pattern analysis.

The magnetism is suppressed in GaP when the implanted Mn concentration is increased or decreased away from the optimum value (as is also seen in material doped during epitaxial growth) or when n-type GaP substrates are used, as shown in Fig. 9. 


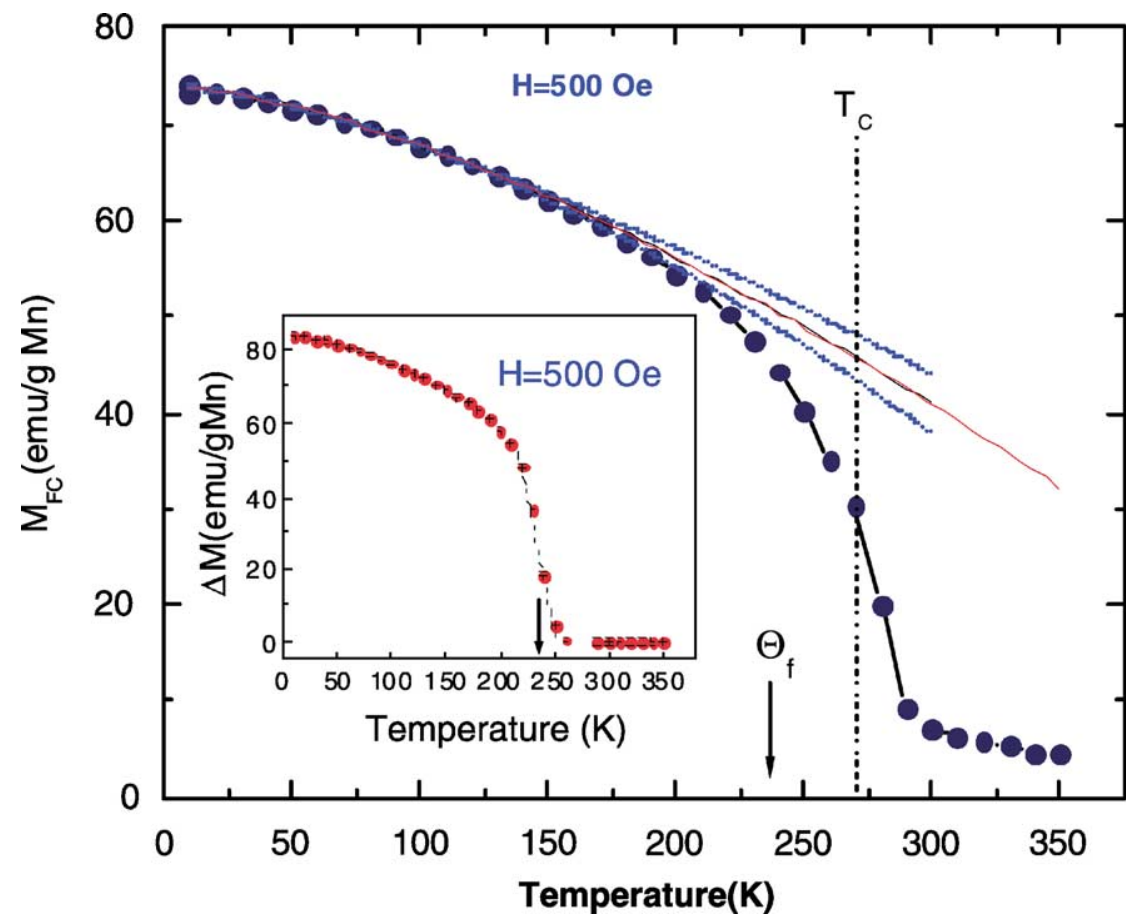

Fig. 7. Field-cooled magnetization of $(\mathrm{Ga}, \mathrm{Mn}) \mathrm{P}$ as a function of temperature. The solid line shows a Bloch law dependence, while the dashed lines are $95 \%$ confidence bands. The vertical dashed line at $T_{\mathrm{C}}=270 \mathrm{~K}$ is the fieldindependent inflection point and the vertical arrows in the main panel and inset mask to ferromagnetic Curie temperature $\Theta_{\mathrm{f}}$. The inset shows the temperature dependence of difference in magnetization between field-cooled and zero-field-cooled conditions.

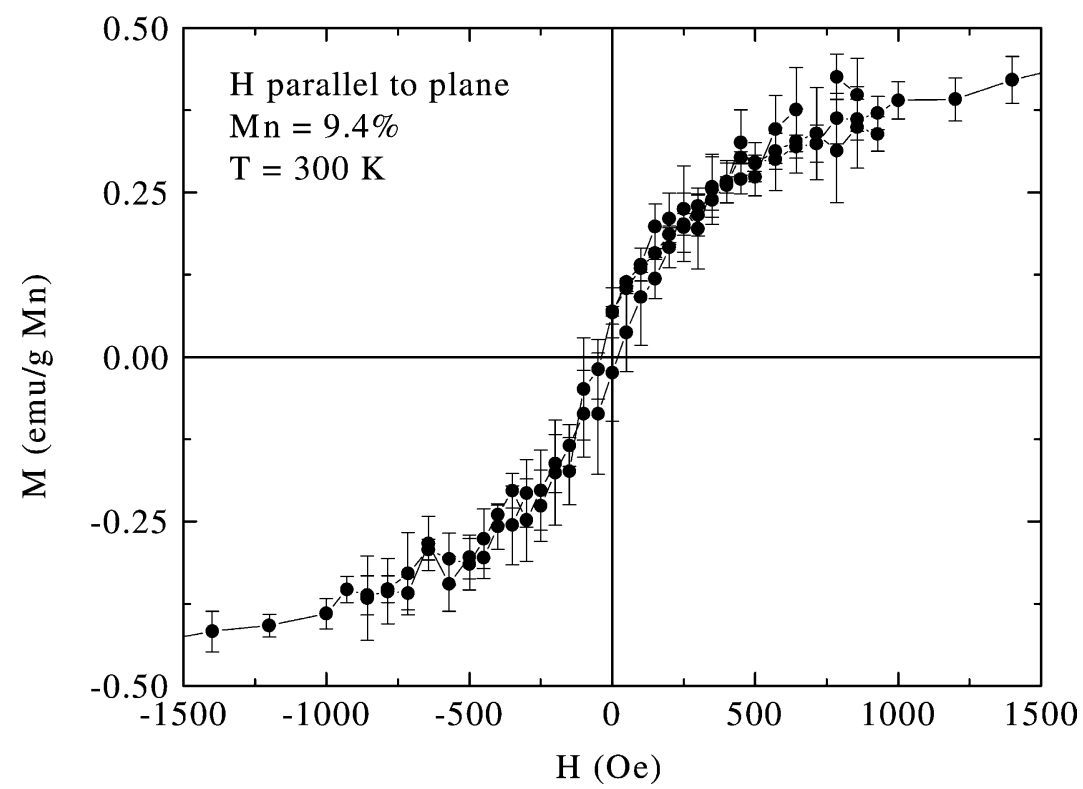

Fig. 8. Room temperature hysteresis loops for epitaxially-grown GaP doped with $9.4 \%$ Mn during growth. 


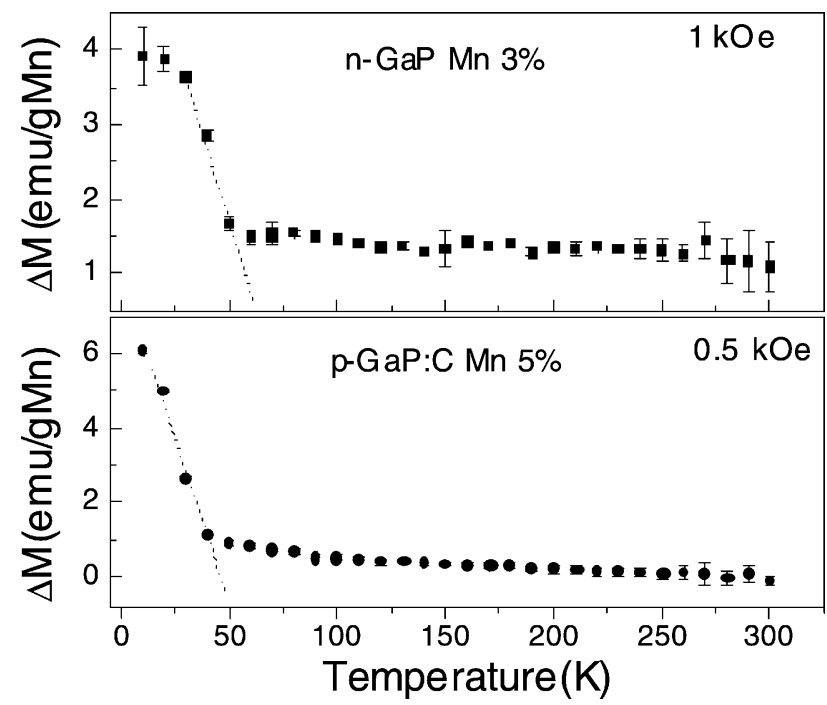

Fig. 9. Temperature dependence of the difference between field-cooled and zero-field-cooled magnetization(per gram $\mathrm{Mn}$ )for n-type $\mathrm{GaP}$ implanted with 3 at.\% Mn (top) or p-type $\mathrm{GaP}$ implanted with 5 at.\% Mn (bottom).

While mean-field theories predict relatively low Curie temperatures $(<110 \mathrm{~K})$ for $(\mathrm{Ga}, \mathrm{Mn}) \mathrm{P}$ [97,98], recent experiments show ferromagnetism above $300 \mathrm{~K}$ [97,98]. In other respects, the magnetic behavior of the ( $\mathrm{Ga}, \mathrm{Mn}) \mathrm{P}$ was consistent with mean-field predictions. For example, the magnetization versus temperature plots showed a more classical concave shape than observed with many DMS materials. In addition, the Curie temperature was strongly influenced by the carrier density and type in the material, with highly p-type samples showing much higher values than n-type or undoped samples. Finally, the Curie temperature increased with Mn concentration up to $\sim 6$ at.\% and decreased at higher concentrations [98]. No secondary phases or clusters could be detected by transmission electron microscopy, X-ray diffraction or selected-area diffraction patterns. Similar results were achieved in samples in which the Mn was incorporated during MBE growth or directly implanted with Mn.

$\mathrm{GaP}$ is a particularly attractive host material for spintronic applications because it is almost lattice-matched to $\mathrm{Si}$. One can therefore envision integration of $(\mathrm{Ga}, \mathrm{Mn}) \mathrm{P}$ spintronic magnetic sensors or data storage elements to form fast non-volatile magnetic random access memories (MRAM). Although it has an indirect bandgap, it can be made to luminescence through addition of isoelectric dopants such as nitrogen or else one could employ the direct bandgap ternary InGaP, which is lattice matched to GaAs. The quaternary InGaAlP materials system is used for visible lightemitting diodes, laser diodes, heterojunction bipolar transistors and high electron mobility transistors. An immediate application of the DMS counterparts to the component binary and ternary materials in this system would be to add spin functionality to all of these devices. A further advantage to the wide bandgap phosphides is that they exhibit room temperature ferromagnetism even for relatively high growth temperatures during MBE.

Obviously, the Mn can also be incorporated during MBE growth of the ( $\mathrm{Ga}, \mathrm{Mn}) \mathrm{P}$. The p-type doping level can be separately controlled by incorporating carbon from a $\mathrm{CBr}_{4}$ source while $\mathrm{P}$ is obtained from thermal cracking of $\mathrm{PH}_{3}$. A phase diagram for the epi growth of this materials system has been developed and this can be used to tailor the magnetic properties of the ( $\mathrm{Ga}, \mathrm{Mn}) \mathrm{P}$. For samples grown at $600 \mathrm{C}$ with 9.4 at.\% Mn, hysteresis is still detectable at $300 \mathrm{~K}$, with a coercive field of $\sim 39$ Oe. 


\section{2. $(\mathrm{Ga}, \mathrm{Mn}) \mathrm{N}$}

The initial work on this material involved either microcrystals synthesized by nitridization of pure metallic $\mathrm{Ga}$ in supercritical ammonia or bulk crystals grown in reactions of $\mathrm{Ga} / \mathrm{Mn}$ alloys on GaN/Mn mixtures with ammonia at $\sim 1200{ }^{\circ} \mathrm{C}$ [99]. These samples exhibit paramagnetic properties over a broad range of Mn concentrations, as did some of its early MBE-grown films.

The first reports of the magnetic properties of $(\mathrm{Ga}, \mathrm{Mn}) \mathrm{N}$ involved bulk microcrystallites grown at high temperatures $\left(\sim 1200{ }^{\circ} \mathrm{C}\right)$, but while percent levels of $\mathrm{Mn}$ were incorporated, the samples exhibited paramagnetic behavior [99]. By sharp contrast, in epitaxial GaN layers grown on sapphire substrates and then subjected to solid state diffusion of $\mathrm{Mn}$ at temperatures from 250 to $800{ }^{\circ} \mathrm{C}$ for various periods, clear signatures of room temperature ferromagnetism were observed [100,101]. Fig. 10 shows anomalous Hall effect data (top) at $323 \mathrm{~K}$ and the temperature dependence of sheet resistance at zero applied field for two different Mn-diffused samples and an undoped GaN control sample (bottom) [101]. The Curie temperature was found to be in the range 220-370 K, depending on the diffusion conditions. The use of ion implantation to introduce the Mn produced lower magnetic ordering temperatures [102].

In $(\mathrm{Ga}, \mathrm{Mn}) \mathrm{N}$ films grown by MBE at temperatures between $580-720^{\circ} \mathrm{C}$ with $\mathrm{Mn}$ contents of 6-9 at.\%, magnetization $(M)$ versus magnetic field $(H)$ curves showed clear hysteresis at $300 \mathrm{~K}$, with coercivities of $52-85 \mathrm{Oe}$ and residual magnetizations of $0.08-0.77 \mathrm{emu} / \mathrm{g}$ at this temperature [103,104]. Fig. 11 shows the temperature dependence of the magnetization for a sample with 9 at.\% $\mathrm{Mn}$, yielding an estimated $T_{\mathrm{C}}$ of $940 \mathrm{~K}$ using a mean-field approximation. Note that while the electrical properties of the samples were not measured, they were almost certainly n-type. As we discussed above, it is difficult to obtain high Curie temperatures in n-type DMS materials according to the mean-field theories and this is something that needs to be addressed in future refinements of these theories. Room temperature ferromagnetism in n-type $(\mathrm{Ga}, \mathrm{Mn}) \mathrm{N}$ grown by MBE has also been reported by Thaler et al. [105]. In that case, strenuous efforts were made to exclude any possible contribution from the sample holder in the superconducting quantum interference device (SQUID) magnetometer or other spurious effects.

Using the optimum growth conditions of $T_{\mathrm{G}}=700{ }^{\circ} \mathrm{C}$ and $\mathrm{Mn}$ cell temperature of $\sim 3$ at. $\%$, no second phases were observed in either high resolution transmission electron microscopy (TEM) or selected-area diffraction patterns (SADP), as shown in Fig. 12.

Fig. 13 shows the result of single axis $\omega-2 \theta$ rocking curve analysis on films grown on both sapphire substrates and MOCVD GaN buffer layers. As with the $\theta-2 \theta$ X-ray diffraction and the results from TEM, the $\omega-2 \theta$ scans do not indicate the presence of second phases within either of the two films. In Fig. 13a, the peak at approximately $34.55^{\circ}$ corresponds to the $\left(\begin{array}{lll}0 & 0 & 2\end{array}\right)$ reflections from the $\mathrm{GaN}$ and $\mathrm{GaMnN}$ layers, while the peak at $41.67^{\circ}$ corresponds to the $\left(\begin{array}{lll}0 & 0 & 6\end{array}\right)$ reflection of sapphire. The small peak at $35.89^{\circ}$ is due to the $\left(\begin{array}{lll}0 & 0\end{array}\right)$ reflection of the AlN buffer layer. Fig. $13 \mathrm{~b}$ again confirms the $\theta-2 \theta$ result for the MOCVD sample, prominently showing only the $\left(\begin{array}{lll}0 & 0 & 2\end{array}\right)$ peak from the $\mathrm{GaN}$ and $\mathrm{GaMnN}$ layers, as well as the $\left(\begin{array}{ll}0 & 0\end{array}\right)$ peak from the substrate. Due to the large FWHM of the underlying thick ( $3 \mu \mathrm{m})$ MOCVD GaN layer (also seen in as-received GaN), features in $2 \theta$ due to the GaMnN ( $\left.\begin{array}{lll}0 & 0 & 2\end{array}\right)$ peak are obscured. The placement of an analyzer crystal in the reflected beam would be required to possibly deconvolute the $\mathrm{GaN}$ peak from the GaMnN peak. The absence of an easily discernible knee on either side of the $\left(\begin{array}{lll}0 & 0\end{array}\right) \mathrm{GaN}$ peak in Fig. 13a as well indicates a low degree of Mn substitution into the Ga sublattice for this film, despite its observed magnetic properties at low temperatures (see Section 4). This is confirmed by the calculated moment per Mn ion, which is found to be approximately $0.3 \mu_{\mathrm{B}}$ per Mn, far less than the $5 \mu_{\mathrm{B}}$ per Mn one would expect for $100 \% \mathrm{Mn}$ substitution. 

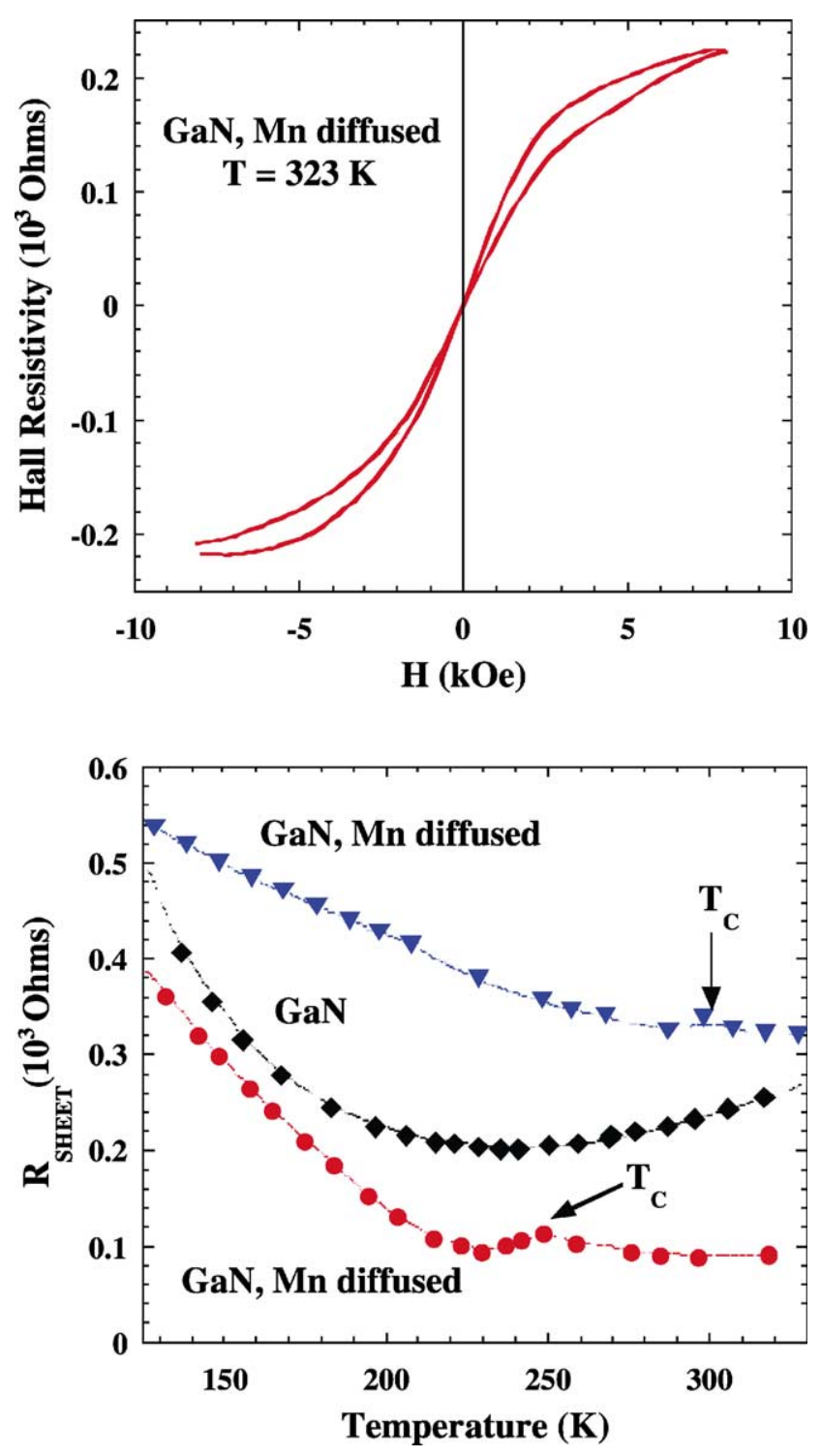

Fig. 10. Temperature dependence of sheet resistance at zero magnetic field for Mn-diffused GaN and as-grown GaN (top) and room temperature anomalous Hall effect hysteresis curves for Mn-diffused GaN (bottom) (after [101]).

Magnetotransport properties of the GaMnN material were investigated in the temperature range between 10 and $300 \mathrm{~K}$ for magnetic field sweeps between -7 and $+7 \mathrm{~T}$. The Hall (transverse) and sheet (longitudinal) resistances were measured after applying In Ohmic contacts to the GaMnN. An ac impedence bridge was used in these measurements to maximize the signal to background noise ratio. The electron carrier density at $300 \mathrm{~K}$ was found to be $2.4 \times 10^{19} \mathrm{~cm}^{-3}$, while the carrier density at $10 \mathrm{~K}$ was found to decrease slightly to $1.3 \times 10^{19} \mathrm{~cm}^{-3}$. The high carrier density at $300 \mathrm{~K}$ indicates that the high growth temperature was effective in increasing the number of free electrons via an excess in nitrogen vacancies, despite the presence of the high concentration of $\mathrm{Mn}$. The sheet resistance showed clear negative magnetoresistance $(11 \%$ at $10 \mathrm{~K})$ as shown in Fig. 14. The anomalous Hall effect was observed up to $\sim 20 \mathrm{~K}$, which was limited by instrumental resolution. The 


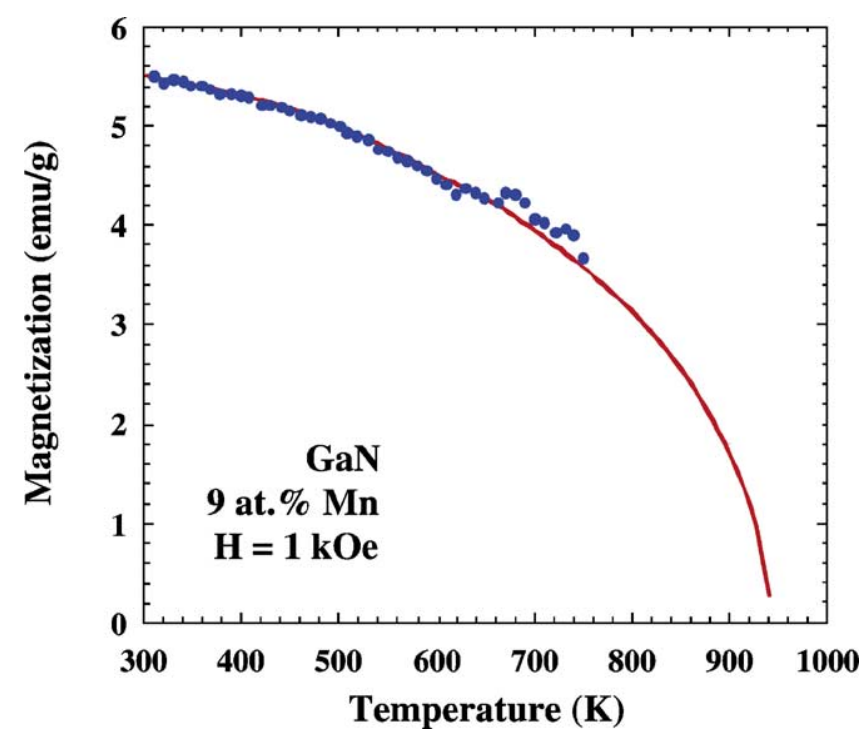

Fig. 11. Magnetization vs. temperature for $(\mathrm{Ga}, \mathrm{Mn}) \mathrm{N}$ sample grown by MBE with $\sim 9$ at. $\% \mathrm{Mn}$. The extrapolation of the curve is based on a mean-field approximation (after $[103,104])$.

Hall resistance data was found to be linear from 25 to $300 \mathrm{~K}$, and is consistent with the absence of magnetic moments due to the thermodynamically stable ferrimagnetic $\mathrm{Mn}_{4} \mathrm{~N}$ phase, which is reported to have a $T_{\mathrm{C}}$ as high as $745 \mathrm{~K}$. In accordance with most of the theoretical predictions, magnetotransport data showed the anomalous Hall effect, negative magnetoresistance and magnetic resistance at temperatures that were dependent on the Mn concentration. For example, in films with very low $(<1 \%)$ or very high $(\sim 9 \%)$ Mn concentrations, the Curie temperatures were between 10 and $25 \mathrm{~K}$. An example is shown in Fig. 15 for an n-type $(\mathrm{Ga}, \mathrm{Mn}) \mathrm{N}$ sample with $\mathrm{Mn} \sim 7 \%$. The sheet resistance shows negative magnetoresistance below $150 \mathrm{~K}$, with the anomalous Hall coefficient disappearing below $25 \mathrm{~K}$. When the Mn concentration was decreased to 3 at.\%, the (Ga, Mn)N showed the highest degree of ordering per Mn atom [105] Fig. 15 shows hysteresis present at $300 \mathrm{~K}$, while the magnetization as a function of temperature is also shown data from samples with different Mn concentrations is shown in Fig. 15 (bottom) and indicates ferromagnetic coupling, leading to a lower moment per Mn. Data from field-cooled and zero-field-cooled conditions was further suggestive of room temperature magnetization [105]. The significance of these results is that there are many advantages from a device viewpoint to having n-type ferromagnetic semiconductors.

It is also worthwhile to point out that for the studies of $(\mathrm{Ga}, \mathrm{Mn}) \mathrm{N}$ showing ferromagnetic ordering by magnetization measurements, a number of materials characterization techniques did not show the presence of any second ferromagnetic phases within detectable limits. In addition, the values of the measured coercivities are relatively small. If indeed there were undetectable amounts of nano-sized clusters, due to geometrical effects, the expected fields at which these clusters would switch magnetically would be expected to be much larger than what has been observed.

Other transition metals dopants have also shown promising behavior in GaN. Recently, several groups have reported above room temperature $T_{\mathrm{C}}$ for $(\mathrm{Ga}, \mathrm{Cr}) \mathrm{N}$. Park et al. reported room temperature ferromagnetism $\left(T_{\mathrm{C}}=280 \mathrm{~K}\right)$ for bulk single crystal $\mathrm{Cr}$-doped $\mathrm{GaN}$ prepared by sodium flux growth method [106]. Epitaxial $(\mathrm{Ga}, \mathrm{Cr}) \mathrm{N}$ prepared by ECR molecular beam epitaxy with $T_{\mathrm{C}}>400 \mathrm{~K}$ was reported by Hashimoto et al. [107]. Ferromagnetic ordering in low temperature-molecular beam epitaxy (LT-MBE) Cr-doped GaAs DMS has also been reported [108]. Although Co-doped $\mathrm{TiO}_{2}$ and $\mathrm{ZnO}$ have reported $T_{\mathrm{C}}$ above $300 \mathrm{~K}$, we are unaware of reports of Co 

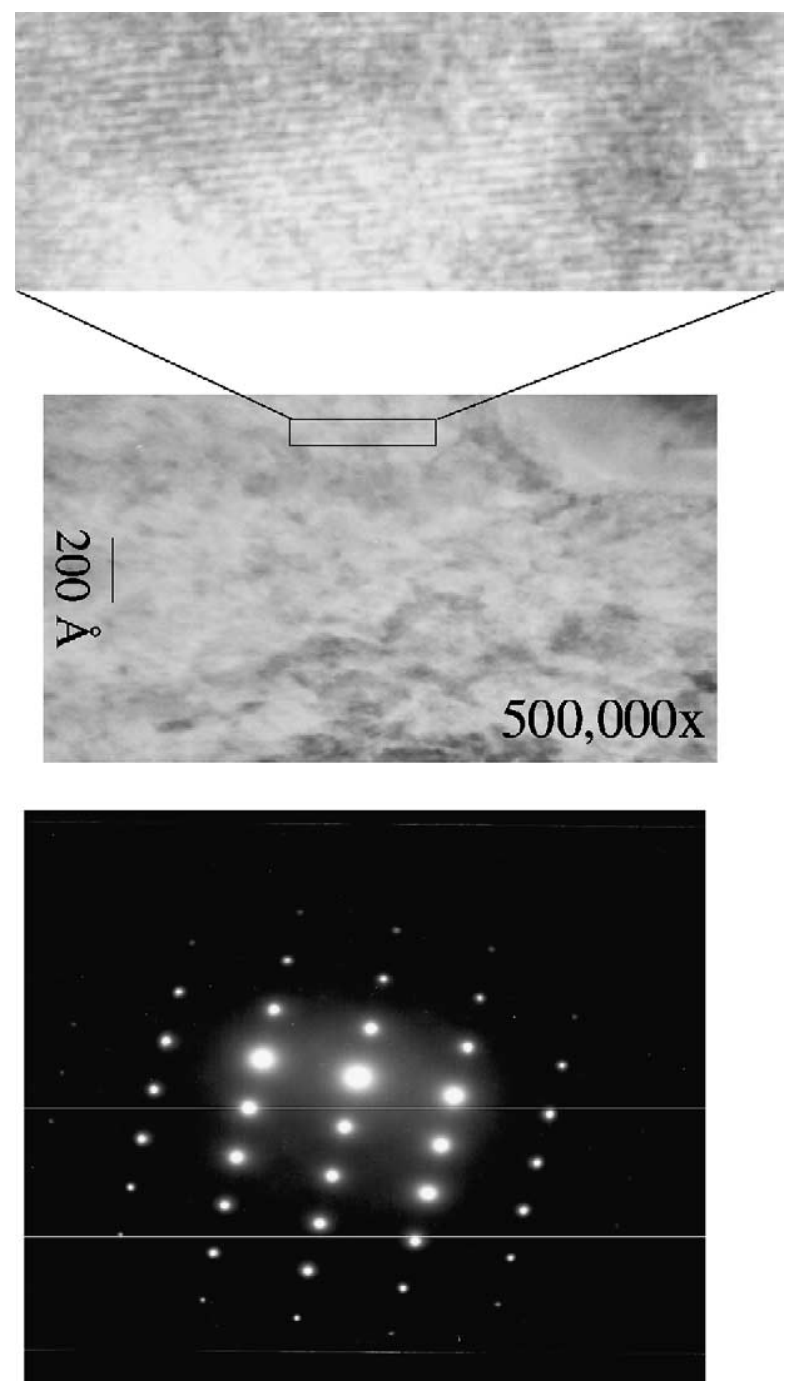

Fig. 12. High resolution TEM (top) and SADP (bottom) of 3 at. $\% \mathrm{Mn} \mathrm{GaMnN}$ grown at $700{ }^{\circ} \mathrm{C}$.

incorporated GaN DMS thus far. For V-doped, we are not aware of any reports of DMS material systems. As in case of all DMS preparation, possible contributions from magnetic second phases are of concern. For example, in the well-studied (Ga, Mn)As DMS system, for high substrate growth conditions, ferromagnetic intermetallic $\left(T_{\mathrm{C}} \sim 300 \mathrm{~K}\right) \mathrm{MnAs}$ clusters form. Binary intermetallic phases for the transition metals $\mathrm{Cr}$, $\mathrm{Co}$, and $\mathrm{V}$ in GaN DMS preparation ranges from superconducting to antiferromagnetic in magnetic behavior. Of note, are $\mathrm{CrN}$ [109] antiferromagnetic which Neél temperature of $273 \mathrm{~K}$ (prepared by MBE and annealing at $800{ }^{\circ} \mathrm{C}$ ) - which is similar to magnetic transition reported by Park et al. (prepared by sodium flux method at $750{ }^{\circ} \mathrm{C}$ ); CoN [110] Pauli paramagnetic for all phases $\left(\mathrm{CoN}, \delta-\mathrm{Co}_{2} \mathrm{~N}\right.$, and $\gamma-\mathrm{Co}_{3} \mathrm{~N}$ prepared by annealing reactive ion sputtering); and ferromagnetic phase $\mathrm{Cr}_{2} \mathrm{~N}$.

Fig. 16 shows the zero-field-cooled and field-cooled magnetization from a Cr-implanted p-GaN sample, showing magnetization still present at $350 \mathrm{~K}$. Magnetization as function of applied field at $5 \mathrm{~K}$ is plotted in Fig. 17 (diamagnetic contributions from the sapphire substrate has been carefully subtracted in all magnetic data) for $\mathrm{Co}, \mathrm{Cr}$ or $\mathrm{V}$-implanted $\mathrm{GaN}$. For $\mathrm{Co}$ and $\mathrm{Cr}$ ion-implanted 

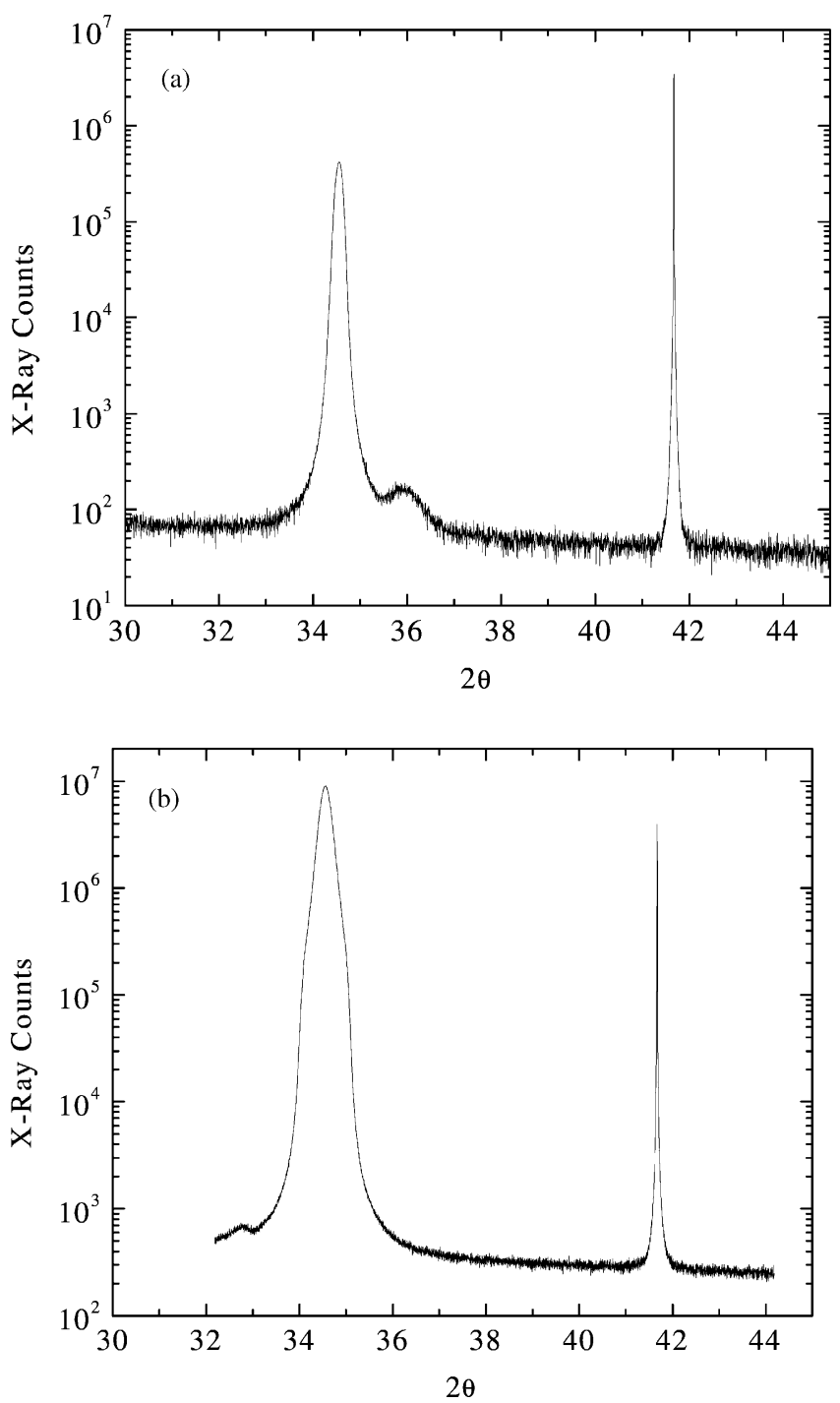

Fig. 13. Single axis $\omega-2 \theta$ rocking curves for GaMnN grown at elevated temperatures on sapphire (a) and MOCVD GaN buffers (b).

samples, ferromagnetic-like loops were observed. For two dosages, magnetic saturation was not observed for applied fields up to $5 \mathrm{~T}$ for both Co and Cr-implanted samples. In other DMS systems with ferromagnetic ordering, for samples which are on the insulating side of the metal-insulator transition, technical saturation of magnetization was found to be difficult. The larger paramagnetic background for higher dose samples is consistent with a carrier mediated model of ferromagnetism in DMS systems at low carrier regimes. Such a material system as wide band gap GaN DMS, at low temperatures, carriers will be localized to the magnetic impurities. If these bound carriers do not overlap with other polarized bound carriers to form a percolation network, ferromagnetic ordering is not maintained. Thus, samples with higher impurity concentrations show greater paramagnetic background as there are more bound carriers to magnetic impurities. If the magnetic properties originate from an undetected ferromagnetic, ferromagnetic, or antiferromagnetic phase, we would expect the higher dose samples to show magnetization approach saturation faster than lower dose 

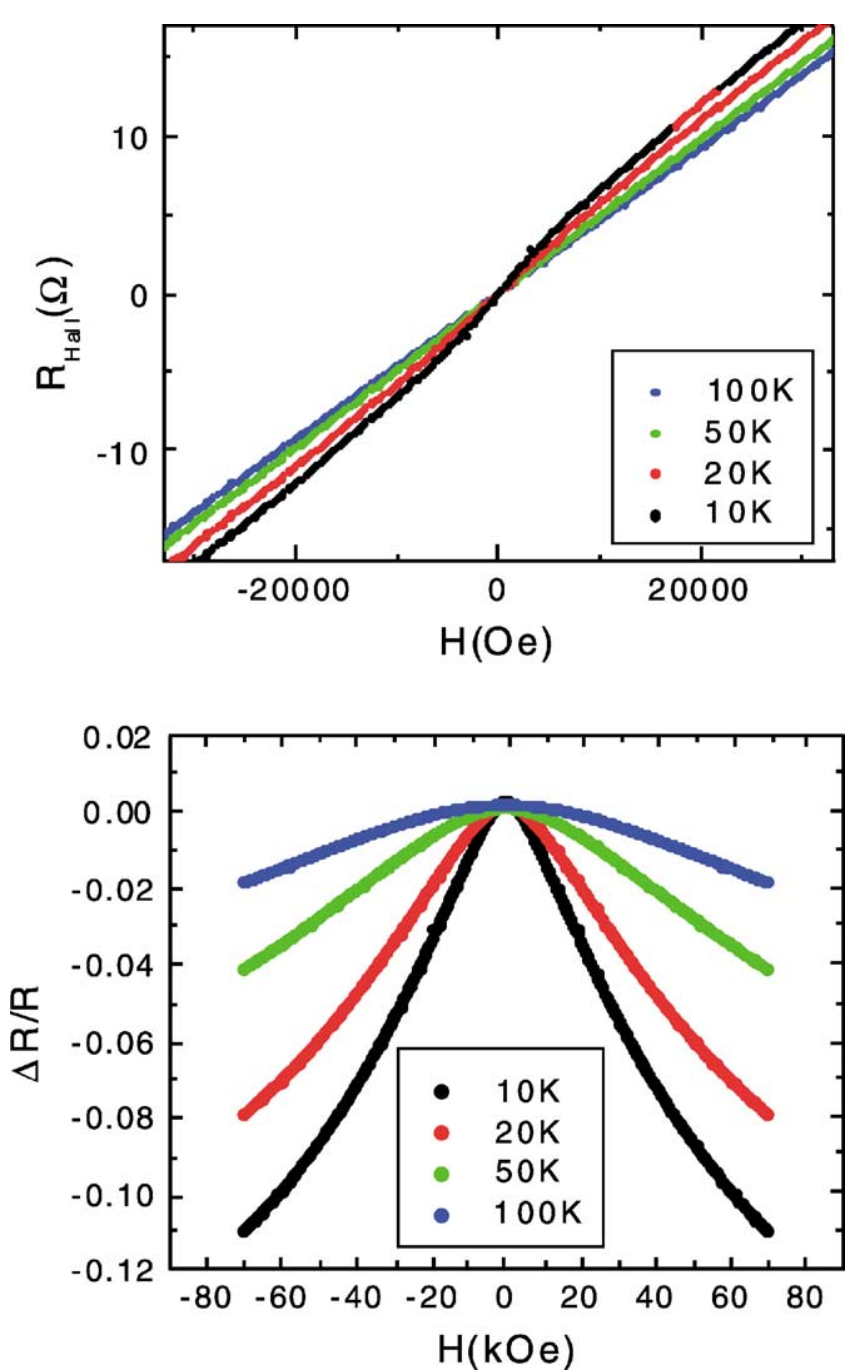

Fig. 14. Magnetotransport data from $\mathrm{GaMnN}$ grown at $T_{\mathrm{G}}=925^{\circ} \mathrm{C}$ on an MOCVD GaN buffer.

samples. For all V-implanted samples, magnetization as a function of applied field measurements indicate the resulting samples to be paramagnetic as magnetic susceptibility was found to be constant.

Extended X-ray absorption fine structure (EXAFS) measurements performed on $(\mathrm{Ga}, \mathrm{Mn}) \mathrm{N}$ samples grown by $\mathrm{MBE}$ on sapphire at temperatures of $400-650{ }^{\circ} \mathrm{C}$ with $\mathrm{Mn}$ concentrations of $\sim 7 \times 10^{20} \mathrm{~cm}^{-3}$ (i.e. slightly over 2 at.\%) are shown in Fig. 18 [111,112]. The similarity of the experimental data with simulated curves for a sample containing this concentration of Mn substituted for $\mathrm{Ga}$ on substitutional lattice positions indicates that $\mathrm{Mn}$ is in fact soluble at these densities. In the samples grown at $650{ }^{\circ} \mathrm{C}, \leq 1$ at. $\%$ of the total amount of Mn was found to be present as Mn clusters. However, at lower growth temperatures $\left(400{ }^{\circ} \mathrm{C}\right)$, the amount of $\mathrm{Mn}$ that could be present as clusters increased up to $\sim 36$ at. $\%$ of the total Mn incorporated. The ionic state of the substitutional Mn was found to be primarily $\mathrm{Mn}$ (II), so that these impurities act as acceptors when substituting for the Ga with valence three. However, when the electrical properties of these samples were measured, they were found to be resistive [111,112]. This result emphasizes how much more needs to be understood concerning the effects of compensation and unintentional doping of $(\mathrm{Ga}, \mathrm{Mn}) \mathrm{N}$, since the EXAFS 

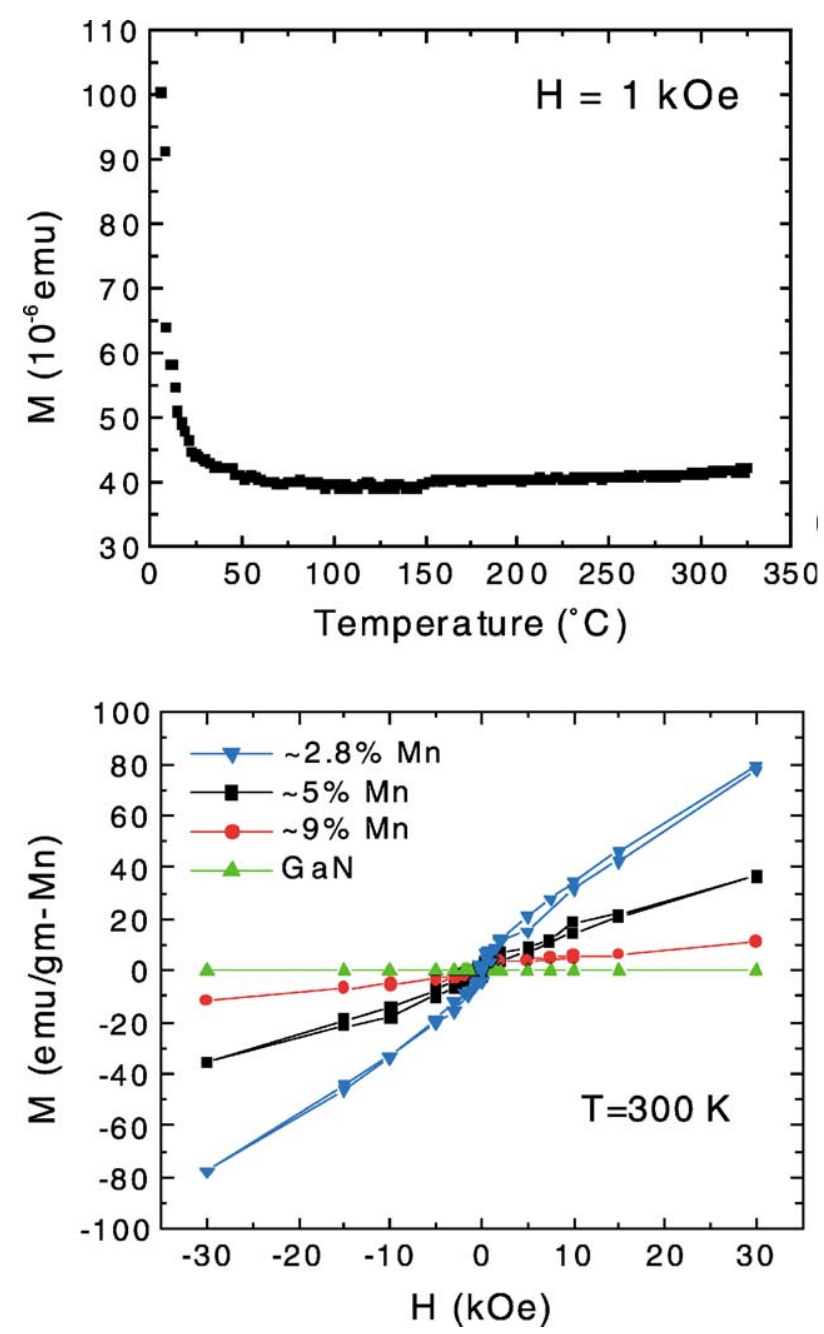

Fig. 15. $M-T$ (top) and $M-H$ (bottom) from GaMnN films grown at $700{ }^{\circ} \mathrm{C}$. The $M-T$ plot is from a sample with $\sim 3$ at. $\%$ $\mathrm{Mn}$.

data indicated the samples should have shown very high p-type conductivity due to incorporation of $\mathrm{Mn}$ acceptors. The local structure and effective chemical valency of $\mathrm{Mn}$ in MBE-grown (Ga, Mn)N samples has been investigated by extended X-ray absorption fine structure [111,112]. It was concluded that most of the Mn was incorporated substitutionally on the Ga sublattice with effective valency close to +2 for samples with $\sim 2$ at. $\% \mathrm{Mn}[111,112]$. There was also evidence that a fraction (from 1 to $36 \%$, depending on growth condition) of the total Mn concentration could be present as small Mn clusters [111,112].

Other reports have also recently appeared on the magnetic properties of $\mathrm{GaN}$ doped with other transition metal impurities. For initially p-type samples directly implanted with either $\mathrm{Fe}$ or $\mathrm{Ni}$,

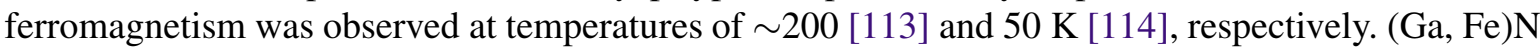
films grown by MBE showed Curie temperatures of $\leq 100 \mathrm{~K}$, with EXAFS data showing that the majority of the Fe was substitutional on $\mathrm{Ga}$ sites [115]. ( $\mathrm{Ga}, \mathrm{Cr}$ )N layers grown in a similar fashion at $700{ }^{\circ} \mathrm{C}$ on sapphire substrates showed single-phase behavior, clear hysteresis and saturation of magnetization at $300 \mathrm{~K}$ and a Curie temperature exceeding $400 \mathrm{~K}$ [116]. 

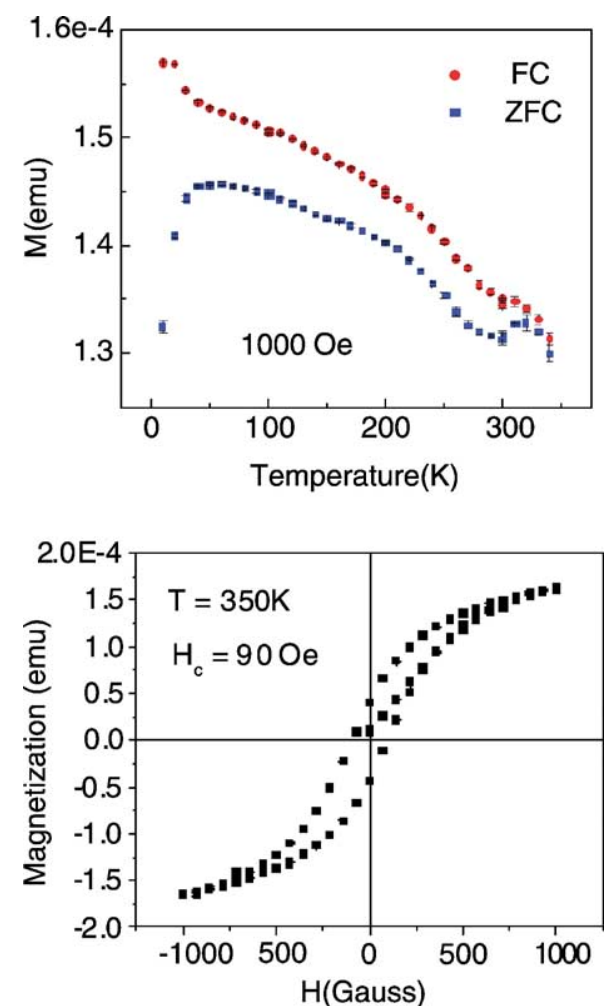

Fig. 16. Field-cooled and zero-field-cooled magnetization for $\mathrm{p}-\mathrm{GaN}$ implanted with 3 at.\% $\mathrm{Cr}$ (top) and hysteresis loop at $350 \mathrm{~K}$ (bottom).

In summary, epi growth of $(\mathrm{Ga}, \mathrm{Mn}) \mathrm{N}$ has produced a range of growth conditions producing single-phase material and the resulting magnetic properties showing ferromagnetism above room temperature [103-105]. In general, no second phases are found for Mn levels below $\sim 10 \%$ for growth temperatures of $\sim 750{ }^{\circ} \mathrm{C}$. The $(\mathrm{Ga}, \mathrm{Mn}) \mathrm{N}$ retains n-type conductivity under these conditions.

\section{3. $\mathrm{ZnO}$}

In addition to Dietl's theory on projected $T_{\mathrm{C}}$ of $\mathrm{Mn}$-doped $\mathrm{ZnO}$, ferromagnetism in magnetically doped $\mathrm{ZnO}$ has been theoretically investigated by ab initio calculations based on local density approximation. Again, the results suggest that ferromagnetic ordering of $\mathrm{Mn}$ is favored when mediated by hole doping. However, for $\mathrm{V}, \mathrm{Cr}, \mathrm{Fe}, \mathrm{Co}$, and $\mathrm{Ni}$ dopants, ferromagnetic ordering in $\mathrm{ZnO}$ is predicted to occur without the need of additional charge carriers [117-121]. Recently, the magnetic properties of $\mathrm{Ni}$-doped $\mathrm{ZnO}$ thin-films were reported [122]. For films doped with 325 at. $\% \mathrm{Ni}$, ferromagnetism was observed at $2 \mathrm{~K}$. Above $30 \mathrm{~K}$, superparamagnetic behavior was observed. In all of these studies, the $\mathrm{ZnO}$ material was n-type. Note that Fukumura et al. have shown that epitaxial thin-films of $\mathrm{Mn}$-doped $\mathrm{ZnO}$ can be obtained by pulsed-laser deposition, with $\mathrm{Mn}$ substitution as high as $35 \%$ while maintaining the wurtzite structure [122]. This is well above the equilibrium solubility limit of $\sim 13 \%$, and illustrates the utility of low-temperature epitaxial growth in achieving metastable solubility in thin-films. Co-doping with $\mathrm{Al}$ resulted in n-type material with carrier concentration in excess of $10^{19} \mathrm{~cm}^{-3}$. Large magnetoresistance was observed in the films, but no evidence for ferromagnetism was reported. Dietl et al. [80] predicted a Curie temperature of 

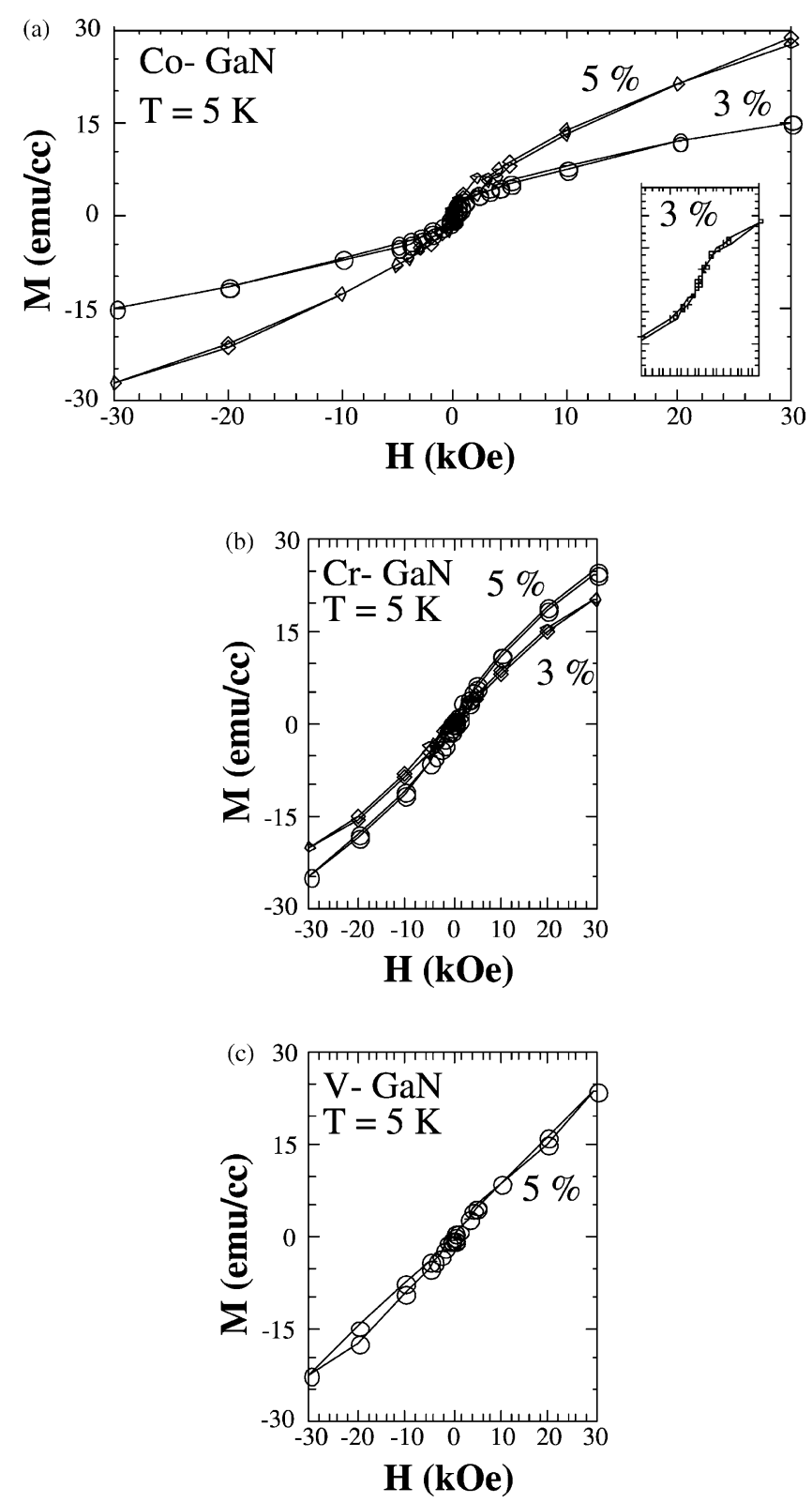

Fig. 17. SQUID magnetometer measurements of magnetization (emu/cc) as a function of applied field (kOe) at $T=5 \mathrm{~K}$ for (a) Co ion-implanted p-GaN/sapphire $\left(\begin{array}{llll}0 & 0 & 1\end{array}\right)$ with $3 \times 10^{15} \mathrm{~cm}^{-2}(\sim 3 \%)$ and $5 \times 10^{15} \mathrm{~cm}^{-2}(\sim 5 \%)$ (inset: $\pm 10 \mathrm{kOe}$ for 3\%); (b) $\mathrm{Cr}$ ion-implanted; (c) V ion-implanted. Only $\mathrm{V}$ ion-implanted samples show a linear relationship between magnetization and applied field.

$\geq 300 \mathrm{~K}$ for $\mathrm{Mn}$-doped $\mathrm{ZnO}$, while electron-doping of $\mathrm{Fe}$, $\mathrm{Co}$ or $\mathrm{Ni}$-doped $\mathrm{ZnO}$ was predicted to stabilize high Curie temperature ferromagnetism [117-121]. Carrier-induced ferromagnetism was predicted for the case of hole doping of $\mathrm{ZnO}(\mathrm{Mn})$ [117-121], while methods for improving p-type doping have also been suggested [120]. Numerous reports of the magnetic properties of transition metal-doped $\mathrm{ZnO}$ have appeared recently [122-126]. Ueda et al. [126] reported Curie temperatures above $300 \mathrm{~K}$ for Co-doped $\mathrm{ZnO}$, Jung et al. [125] a $T_{\mathrm{C}}$ of $45 \mathrm{~K}$ for Mn-doped $\mathrm{ZnO}$ and Wakano et al. [123] a $T_{\mathrm{C}}$ of $\sim 2 \mathrm{~K}$ for Ni-doped $\mathrm{ZnO}$. 


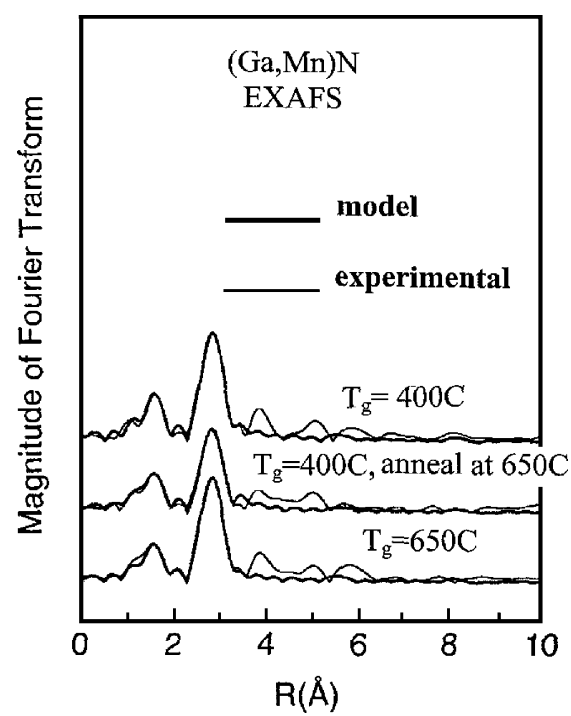

Fig. 18. Fourier transforms of the Mn K-edge EXAFS data from Mn-doped (GaMn)N. The fine lines are the experimental data in these curves, while the course lines are the calculated curves assuming 2 at.\% of $\mathrm{Mn}$ in the GaN (after [111,112]).

The theory by Dietl et al. predicting high temperature ferromagnetism for Mn-doped $\mathrm{ZnO}$ is specifically for p-type material. Experimental results reported in the literature on $\mathrm{Mn}$-doped $\mathrm{ZnO}$ that is n-type due to group III donor impurities are consistent with this prediction, showing no ferromagnetism. However, we recently obtained preliminary evidence for ferromagnetism in n-type $\mathrm{ZnO}$, in which $\mathrm{Mn}$ and Sn serve as the transition metal and donor impurities, respectively. In these experiments, $\mathrm{Mn}$ ions were implanted at elevated temperature into Sn-doped $\mathrm{ZnO}$ single crystals. Magnetization measurements show clear hysteresis in the $B$ versus $H$ curves. Fig. 19 shows the magnetization behavior at $10 \mathrm{~K}$ for a Sn-doped $\mathrm{ZnO}$ sample implanted with 3 at.\% Mn, clearly indicating ferromagnetism. More effort is needed to determine the origin of ferromagnetism in these materials. If carrier-mediated mechanisms are responsible, one must explain why the behavior depends on the specific cation dopant specie chosen ( $\mathrm{Sn}$ versus $\mathrm{Al}, \mathrm{Ga}$ ). Additional work is needed in order to address these issues.

Co-implanted $\mathrm{ZnO}$ has also shown room temperature ferromagnetism, but in this case, the origin of the magnetism is in the presence of oriented nano-crsytals of Co, as seen in the XRD scan in Fig. 20.

It has recently been reported that anatase $\left(\mathrm{TiO}_{2}\right)$ doped with $\mathrm{Co}$ is a ferromagnetic semiconductor $[127,128] . \mathrm{TiO}_{2}$ occurs in three distinct polymorphs, namely rutile, anatase, and brookite. Rutile is the thermodynamically stable phase at high temperature, and is the most widely studied. Anatase is metastable, but can be stabilized in thin-film form. Undoped rutile is an anisotropic, tetragonal insulator ( $a=4.59 \AA, c=2.96 \AA$ ) possessing a bandgap of $\sim 3 \mathrm{eV}$. Anatase is also tetragonal ( $a=3.78 \AA, c=9.52 \AA$ ) with a bandgap of $3.2 \mathrm{eV} \mathrm{[129]} \mathrm{At} \mathrm{low} \mathrm{temperatures,} \mathrm{the}$ permittivity of rutile is $\sim 110$ along the $a-b$-direction and $\sim 240$ along the $c$-axis [130] The static dielectric constant of anatase is 31 [131]. $\mathrm{TiO}_{2}$ can be made an n-type semiconductor with $\mathrm{n} \sim 10^{19} \mathrm{~cm}^{-3}$ via cation substitution or by Ti interstitials Low temperature electron Hall mobility on the order of $30-100 \mathrm{~cm}^{2} /(\mathrm{V} \mathrm{s})$ has been reported for rutile. Hall mobility of electron-doped anatase has been measured as high as $20 \mathrm{~cm}^{2} /(\mathrm{V} \mathrm{s})$.

Other reports have shown clear evidence for Co nanoclusters as the cause of room temperature ferromagnetism in anatase $\mathrm{Ti}_{0.96} \mathrm{Co}_{0.04} \mathrm{O}$ thin-films grown on $\mathrm{SrTiO}_{2}$ substrates by pulsed-laser deposition [132]. At this stage, it is unclear whether this is a general result. 

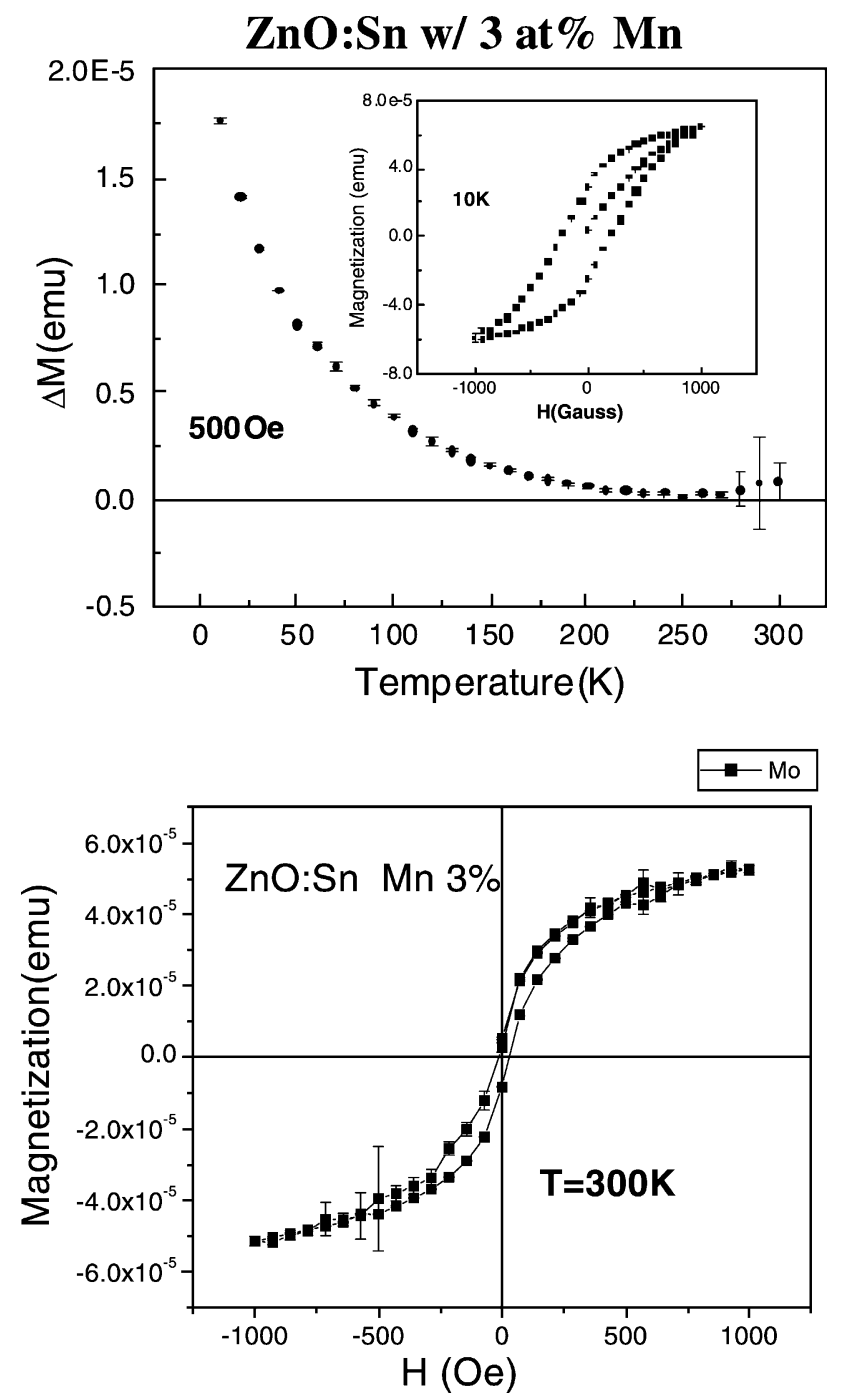

Fig. 19. Temperature dependence of difference in field-cooled and zero-field-cooled magnetization for bulk $\mathrm{ZnO}(\mathrm{Sn})$ implanted with 3 at.\% Mn (top) and room temperature hysteresis from same sample (bottom).

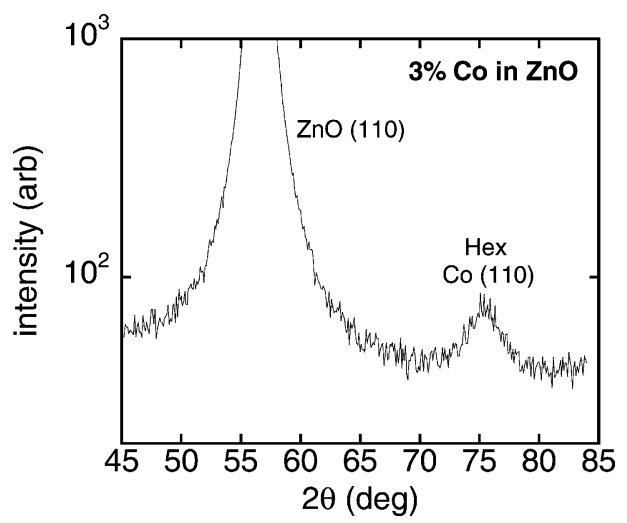

Fig. 20. XRD scans of Co-implanted ( 3 at. \%) bulk $\mathrm{ZnO}$ after annealing at $700{ }^{\circ} \mathrm{C}$. Peaks due to Co clusters are present. 


\subsection{Chalcopyrite materials}

The chalcopyrite semiconductors are of interest for a number of applications. For example, $\mathrm{ZnGeP}_{2}$ exhibits unusual non-linear optical properties and can be used in optical oscillators and frequency converters. $\mathrm{ZnSnAs}_{2}$ shows promise for far-IR generation and frequency converters. The wide bandgap chalcopyrites $\mathrm{ZnGeN}_{2}$ and $\mathrm{ZnSiN}_{2}$ have lattice parameters close to $\mathrm{GaN}$ and $\mathrm{SiC}$, respectively, and the achievement of ferromagnetism in these materials would make it possible for direct integration of magnetic sensors and switches with blue/green/UV lasers and light-emitting diodes, UV solar-blind detectors and microwave power electronic devices fabricated in the $\mathrm{GaN}$ and $\mathrm{SiC}$. The bandgap of $\mathrm{ZnGe}_{x} \mathrm{Si}_{1-x} \mathrm{~N}_{2}$ varies linearly with composition from $3.2(x=1)$ to $4.46 \mathrm{eV}$ $(x=0)$.

Numerous reports of room temperature ferromagnetism in Mn-doped chalcopyrites have appeared. A compilation of these results and those from transition metal-doped GaN and GaP are shown in Tables 1 and 2. The $\mathrm{ZnSnAs}_{2}$ is somewhat of an anomaly due to its small bandgap, but little theory is available at this point on the chalcopyrites and their expected magnetic properties as a function of bandgap, doping or Mn concentration. In the only case in which electrical properties were reported, the $\mathrm{ZnGeSiN}_{2}: \mathrm{Mn}$ was n-type [133]. Fig. 21 shows $M-T$ and $M-H$ data from an epi $\mathrm{ZnSiGeN}_{2}$ sample grown on a sapphire substrate and implanted with 5 at.\% of $\mathrm{Mn}$. The magnetization persists to $300 \mathrm{~K}$, according to the $M-T$ plot and no second phases were detected by TEM or XRD [133]. The state of development of the chalcopyrite materials is still at a very early stage and basic information is still not available on parameters such as the energy level of $\mathrm{Mn}$ in the bandgap.

\section{Potential device applications}

Previous articles have discussed some spintronic device concepts such as spin junction diodes and solar cells [9], optical isolators and electrically-controlled ferromagnets [10]. The realization of light-emitting diodes with a degree of polarized output has been used to measure spin injection

Table 1

Compilation of semiconductors and oxides showing room temperature ferromagnetism

\begin{tabular}{|c|c|c|c|c|}
\hline Material & $\begin{array}{l}\text { Bandgap } \\
(\mathrm{eV})\end{array}$ & Comments & $\begin{array}{l}\text { Ordering } \\
\text { temperature }(\mathrm{K})\end{array}$ & Reference \\
\hline $\mathrm{Cd}_{1-x} \mathrm{Mn}_{x} \mathrm{GeP}_{2}$ & 1.72 & Solid-phase reaction of evaporated $\mathrm{Mn}$ & $>300$ & [74] \\
\hline$(\mathrm{Ga}, \mathrm{Mn}) \mathrm{N}$ & 3.4 & Mn incorporated by diffraction & $228-370$ & {$[100,101]$} \\
\hline$(\mathrm{Ga}, \mathrm{Mn}) \mathrm{N}$ & 3.4 & Mn incorporated during MBE; n-type & $>300$ & {$[105]$} \\
\hline$(\mathrm{Ga}, \mathrm{Mn}) \mathrm{N}$ & 3.4 & Mn incorporated during MBE & $940^{\mathrm{a}}$ & {$[103,104]$} \\
\hline$(\mathrm{Ga}, \mathrm{Cr}) \mathrm{N}$ & 3.4 & $\mathrm{Cr}$ incorporated during $\mathrm{MBE}$ & $>400$ & {$[107]$} \\
\hline$(\mathrm{Ga}, \mathrm{Cr}) \mathrm{N}$ & 3.4 & Bulk growth & $\sim 280$ & [106] \\
\hline$(\mathrm{Ga}, \mathrm{Gd}) \mathrm{N}$ & 3.4 & Gd incorporated during $\mathrm{MBE}$ & $>400$ & {$[153]$} \\
\hline$(\mathrm{Al}, \mathrm{Cr}) \mathrm{N}$ & 6.2 & Reactive co-sputtering & $>340$ & {$[155]$} \\
\hline$(\mathrm{ZnO}): \mathrm{Co}$ & $3.1-3.6$ & Co incorporated during PLD; $\sim 15 \%$ Co & $>300$ & [122] \\
\hline$\left(\mathrm{TiO}_{2}\right): \mathrm{Co}$ & 3.1 & Anatase; $\sim 7 \% \mathrm{Co} ; \mathrm{PLD}$ or $\mathrm{O}_{2} \mathrm{MBE}$ & $>380$ & {$[127,128]$} \\
\hline$(\mathrm{Ga}, \mathrm{Mn}) \mathrm{P}: \mathrm{C}$ & 2.2 & Mn incorporated by implant or MBE; $\mathrm{p} \sim 10^{20} \mathrm{~cm}^{-3}$ & $>330$ & {$[97,98]$} \\
\hline$\left(\mathrm{Zn}_{1-x} \mathrm{Mn}_{x}\right) \mathrm{GeP}_{2}$ & $1.83-2.8$ & Sealed ampule growth; insulating; $5.6 \% \mathrm{Mn}$ & 312 & [73] \\
\hline$(\mathrm{Zn}, \mathrm{Mn}) \mathrm{GeP}_{2}$ & $<2.8$ & Mn incorporated by diffusion & 350 & [74] \\
\hline $\mathrm{ZnSnAs}_{2}$ & 0.65 & bulk growth & 329 & [76] \\
\hline $\mathrm{ZnSiGeN}_{2}$ & 3.52 & Mn-implanted epi & $\sim 300$ & [133] \\
\hline
\end{tabular}

\footnotetext{
${ }^{\mathrm{a}}$ Extrapolated from measurements up to $\sim 750 \mathrm{~K}$.
} 
Table 2

Compilation of potential second phases in transition metal-doped wide bandgap semiconductors with known magnetic properties

\begin{tabular}{|c|c|c|}
\hline Phase & Nature of magnetism & Applicable magnetic temperature $(\mathrm{K})$ \\
\hline $\mathrm{Co}$ & Ferromagnetic & 1382 \\
\hline $\mathrm{Cr}$ & Antiferromagnetic & 311 \\
\hline $\mathrm{Fe}$ & Ferromagnetic & 1040 \\
\hline $\mathrm{Ni}$ & Ferromagnetic & 627 \\
\hline $\mathrm{Mn}$ & Antiferromagnetic & 100 \\
\hline $\mathrm{Fe}_{3} \mathrm{Ga}_{4}$ & Ferromagnetic & 483 or 697 \\
\hline $\mathrm{Fe}_{2} \mathrm{Ga}$ & Ferromagnetic & 620 \\
\hline $\mathrm{Fe}_{3} \mathrm{Ga}$ & Ferromagnetic & 760 \\
\hline $\mathrm{Fe} / \mathrm{Ga}$ alloys & Ferromagnetic & \\
\hline $\mathrm{Fe}_{4} \mathrm{~N}$ & Ferromagnetic & 760 \\
\hline $\mathrm{FeP}$ & Ferromagnetic & 215 \\
\hline $\mathrm{Fe}_{2} \mathrm{P}$ & Ferromagnetic & 278 \\
\hline $\mathrm{Fe}_{3} \mathrm{P}$ & Ferromagnetic & 716 \\
\hline $\mathrm{FeP}_{2}$ & Antiferromagnetic & 250 \\
\hline $\mathrm{Mn}_{2} \mathrm{Ga}$ & Ferromagnetic & 690 \\
\hline$\varepsilon-\mathrm{Mn}_{3} \mathrm{Ga}$ & Ferromagnetic & 743 \\
\hline$\varsigma-\mathrm{Mn}_{5} \mathrm{Ga}_{8}\left(\mathrm{Mn}_{0.6} \mathrm{Ga}_{0.4}\right)$ & Ferromagnetic & 210 \\
\hline $\mathrm{MnGa}$ & Ferromagnetic & $>300$ \\
\hline $\mathrm{Mn}_{4} \mathrm{~N}$ & Ferromagnetic & 745 \\
\hline $\mathrm{MnP}$ & Ferromagnetic & 291 \\
\hline $\mathrm{MnP}$ & Antiferromagnetic & 50 \\
\hline $\mathrm{Mn}_{3} \mathrm{P}$ & Antiferromagnetic & 115 \\
\hline $\mathrm{Mn}_{2} \mathrm{P}$ & Antiferromagnetic & 103 \\
\hline $\mathrm{CrN}$ & Antiferromagnetic & 273 \\
\hline $\mathrm{Cr}_{2} \mathrm{~N}$ & Ferromagnetic (?) & Not ferromagnetic between \\
\hline $\mathrm{Ni}_{3} \mathrm{P}$ & Pauli paramagnetic & 85 and $500 \mathrm{~K}$ \\
\hline $\mathrm{Ni}_{2} \mathrm{P}$ & Pauli paramagnetic & \\
\hline $\mathrm{NiP}_{2}$ & Exhibits magnetism & \\
\hline Amorphous Ni/P alloys & Weak homogenous ferromagnetism & \\
\hline $\mathrm{CoP}_{2}$ & Diamagnetic semiconductor & \\
\hline $\mathrm{CoP}$ & Weak ferromagnetic & $\ll 1382$ \\
\hline $\mathrm{V}_{3} \mathrm{Ga}$ & Superconductor & $T_{\text {critical }}=16.8 \mathrm{~K}$ \\
\hline
\end{tabular}

efficiency in heterostructures [134-142]. Such structures can reveal much about spin transport through heterointerfaces after realistic device processing schemes involving etching, annealing and metallization. The spin transfer in such situations has proven surprisingly robust [143]. It is obviously desirable that spintronic devices are operable at or above room temperature. As an initial demonstration that $(\mathrm{Ga}, \mathrm{Mn}) \mathrm{N}$ layers can be used as the n-type injection layer in $\mathrm{GaN} / \mathrm{InGaN}$ blue light-emitting diodes, Fig. 22 shows the LED structure and the spectral output. It is necessary to next establish the extent of any degree of polarization of the light emission, which might be difficult to observe in GaN/InGaN LEDs, since it has been shown that the free exciton components in the EL spectrum contribute mostly to the observed circular polarization of the emitted light [144]. While the expected advantages of spin-based devices include non-volatility, higher integration densities, lower power operation and higher switching speeds, there are many factors still to consider in whether any of these can be realized. These factors include whether the signal sizes due to spin effects are large enough at room temperature to justify the extra development work needed to make spintronic devices and whether the expected added functionality possible will materialize. Another example of a potential device application is shown in Fig. 23, which shows a schematic of a ZnO-based, photoinduced ferromagnet grown on a GaAs substrate. 

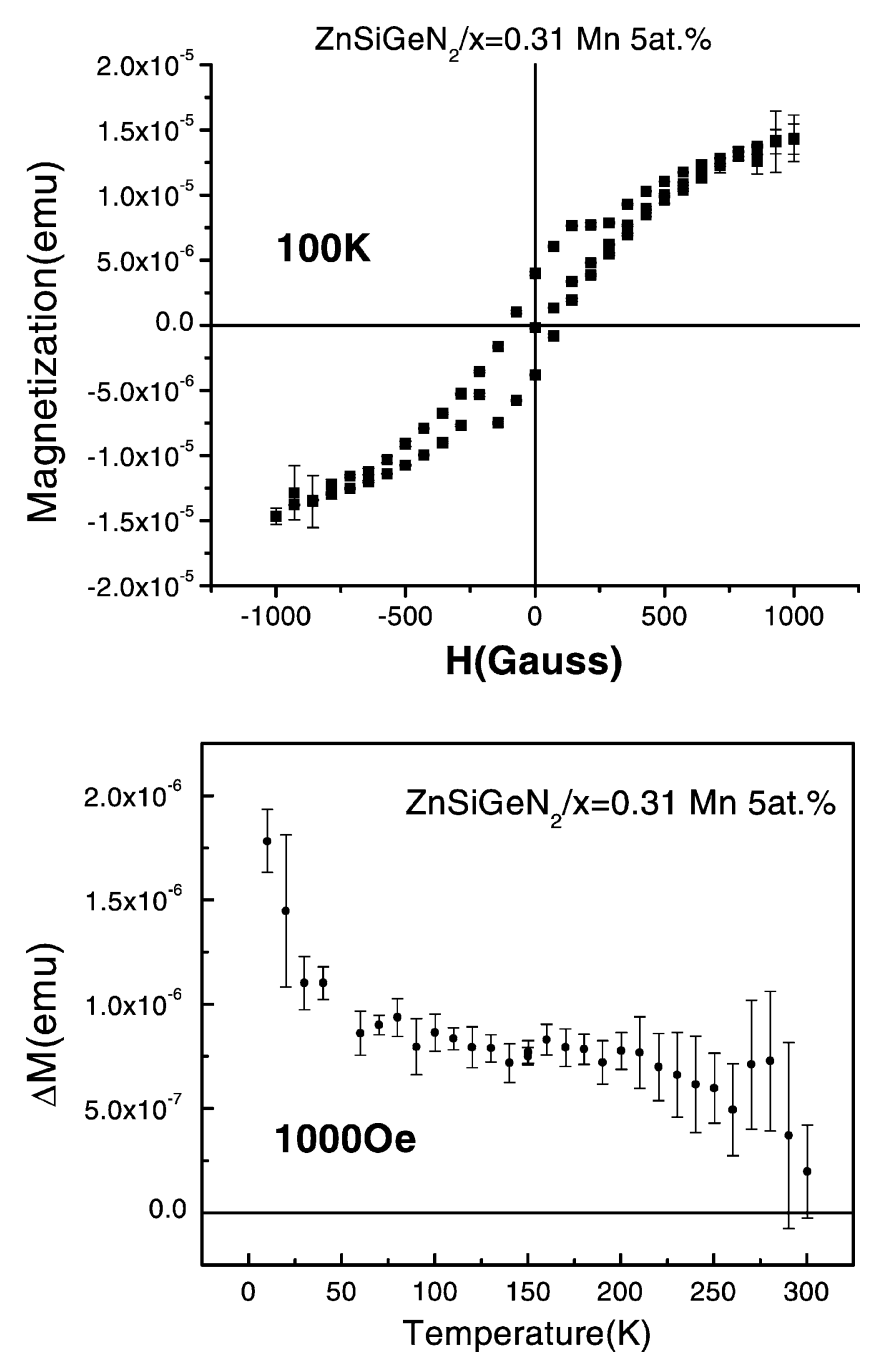

Fig. 21. $M-H$ (top) and $M-T$ (bottom) from $\mathrm{ZnSiGeN}_{2}$ implanted with 5 at.\% $\mathrm{Mn}$ and annealed at $700{ }^{\circ} \mathrm{C}$ (after [133]).

The most commonly pursued device has been the Dutta-Das spinfet, in which gate bias applied to the channel of a field effect transistor would cause precession of spin-polarized current moving from the source to drain. The spin injection could come from polarized metal Ohmic contacts or from DMS injection regions. Fig. 24 shows a potential embodiment of a Si-based spinfet, in which lattice-matched, regrown $\mathrm{p}-\mathrm{GaMnP}$ source and drain regions would be employed for spin injection. This device would be attractive from the viewpoint of integration with existing Si technology.

Other examples of GaMnN-based DMS devices are shown in Fig. 25. The top of the figure shows a spinfet, operating on the same principle as that in Fig. 24, while the bottom of the figure shows an optical rotator. In this device, bias applied to the contacts would deplete the carriers and remove the spin polarization. Polarized incident light would be reflected at a different angle in the two cases.

In addition to active and/or optical devices, wide bandgap DMS materials may also be used as passive devices. LeClair et al. [145] have recently shown an artificial half-metallic structure by using a polycrystalline sputtered ferromagnetic semiconductor $(\mathrm{EuS})$ as a tunneling barrier. This barrier can function as an effective spin filter, since a tunneling electron encounters a differing barrier height 

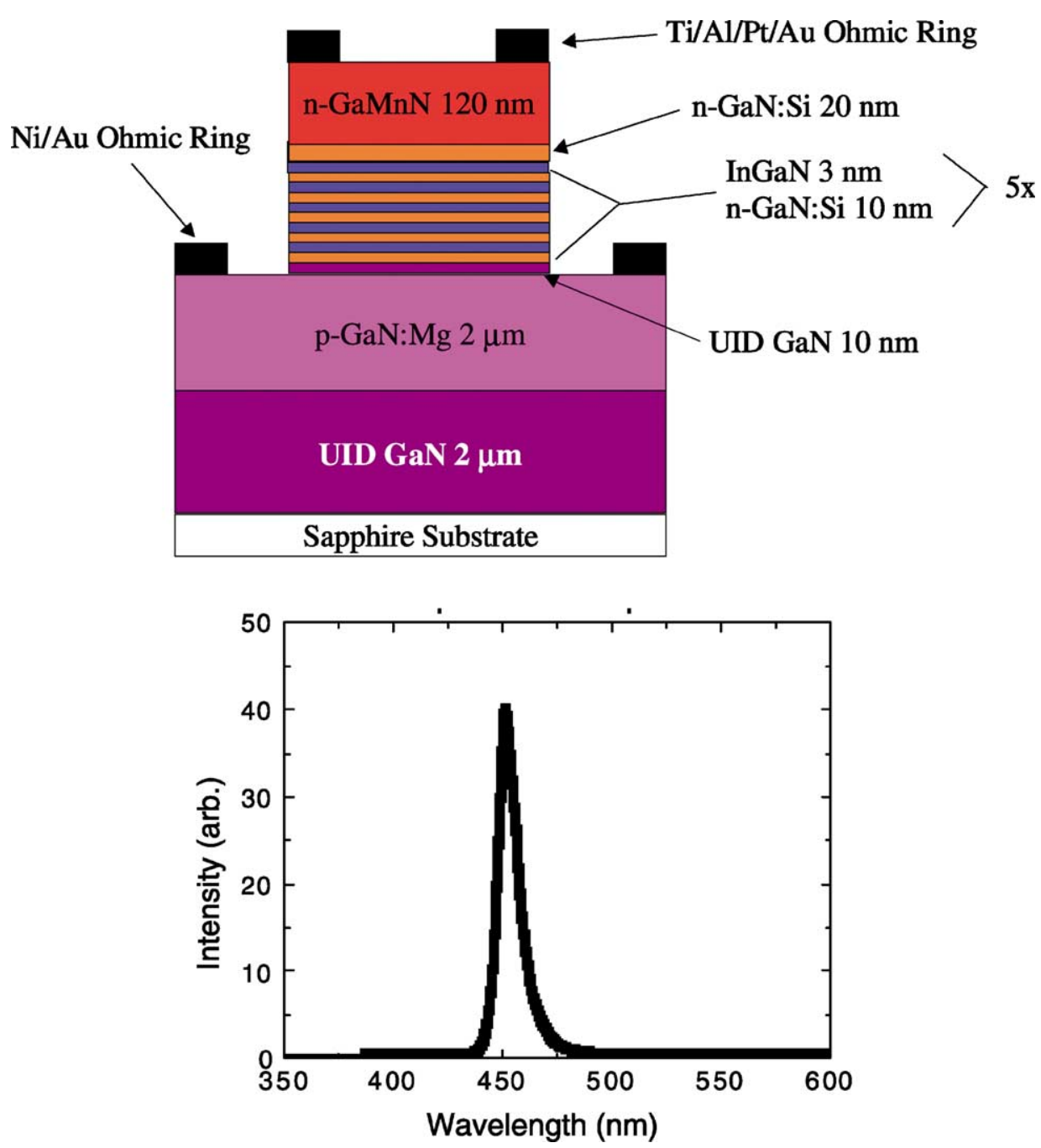

Fig. 22. Schematic (top) and spectral (bottom) output from GaMnN/InGaN light-emitting diode.

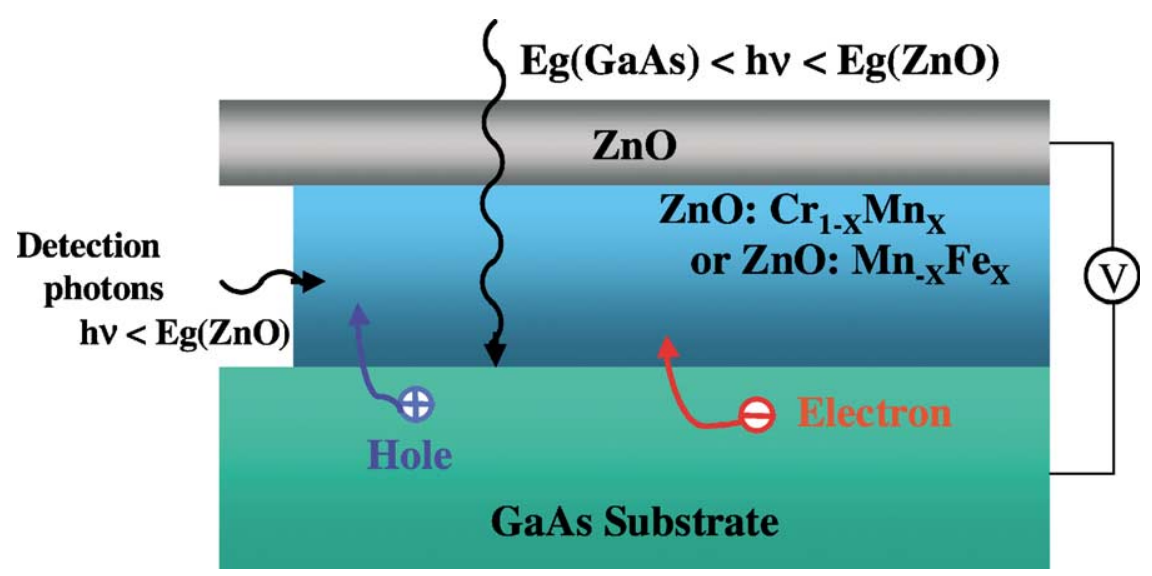

Fig. 23. Schematic of photo-induced, ZnO-based DMS ferromagnet. 


\section{Spin FET on silicon \\ (Datta-Das Configuration)}

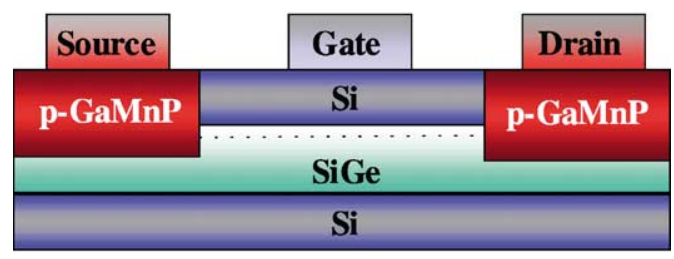

$\mathrm{N}$-type version possible using strained $\mathrm{Si}$ on SiGe strain relief buffer

Fig. 24. Proposed Si-based spinfet grown on a Si substrate and employing regrown p-GaMnP Ohmic contact regions as spin injectors.

depending on its spin below $T_{\mathrm{C}}$ of the barrier material (for EuS, $T_{\mathrm{C}} \sim 16.8 \mathrm{~K}$ ). At low temperatures, the spin-filtering efficiencies were found to be $\sim 90 \%$. Room temperature DMS materials for these spin-filtering effects could be used to increase magnetoresistance changes in current magnetic tunnel junctions and metallic spin-valve structures.
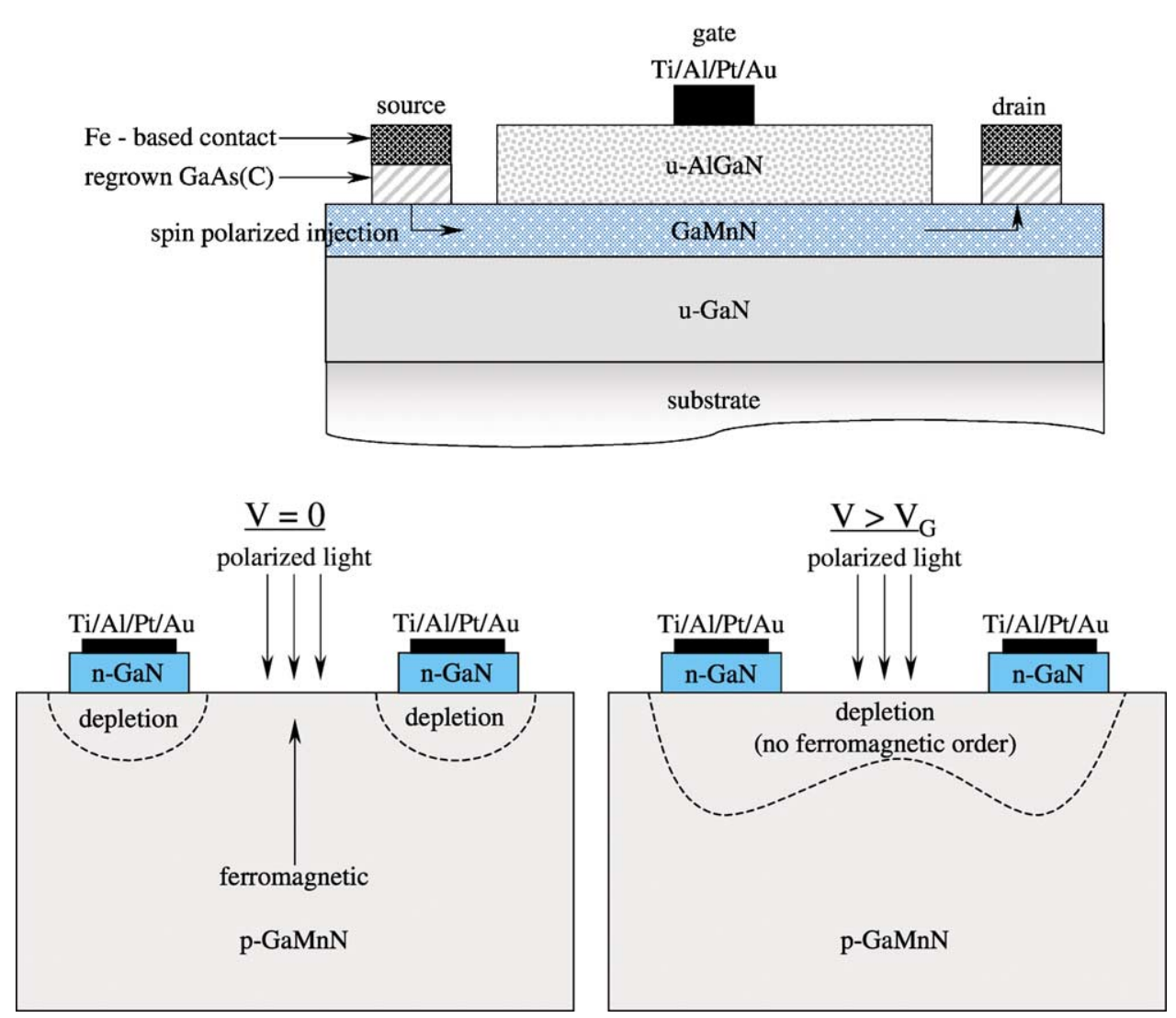

Fig. 25. Schematic of some GaN-based spin devices. At top is a Dutta-Das configuration spinfet and at bottom is an optical rotator. 


\section{Future research}

As described earlier, there are a number of existing models for the observed ferromagnetism in semiconductors. The near-field models consider the ferromagnetism to be mediated by delocalized or weakly localized holes in the p-type materials. The magnetic Mn ion provides a localized spin and acts as an acceptor in most III-V semiconductors so that it can also provide holes. In these models, the $T_{\mathrm{C}}$ is proportional to the density of $\mathrm{Mn}$ ions and the hole density. Many aspects of the experimental data can be explained by the basic mean-field model. However, ferromagnetism has been observed in samples that have very low hole concentrations, in insulating material and more recently in n-type material. Models in these regimes are starting to appear [146-154].

An alternative approach using local density functional calculations suggests that the magnetic impurities may form small nano-size clusters that produce the observed ferromagnetism [82]. These clusters would be difficult to detect by most characterization techniques. Clearly there is a need to more fully characterize the materials showing room temperature ferromagnetism and correlate these results to establish on a case-by-case basis which is the operative mechanism and also to refine the theories based on experimental input. More work is also needed to establish the energy levels of the Mn, whether there are more effective magnetic dopant atoms and how the magnetic properties are influenced by carrier density and type. Even basic measurements such as how the bandgap changes with Mn concentration in $\mathrm{GaN}$ and $\mathrm{GaP}$ have not been performed. The control of spin injection and manipulation of spin transport by external means such as voltage from a gate contact or magnetic fields from adjacent current lines or ferromagnetic contacts is at the heart of whether spintronics can be exploited in device structures and these areas are still in their infancy. A concerted effort on the physics and materials science of the new dilute magnetic semiconductors is underway in many groups around the world, but fresh insights, theories and characterization methods would greatly accelerate the process.

\section{Acknowledgements}

The work at UF was partially supported by NSF-DMR 0101438, NSF-DMR 0101856, ARODAAD 190210420 while the work at SNU was partially supported by KOSEF and Samsung Electronics Endowment through CSCMR and by the Seoul National University Research Foundation. The authors are very grateful to their collaborators M.E. Overberg, G.T. Thaler, R. Frazier, F. Ren, Jihyun Kim, N.A. Theodoropoulou, R. Rairigh, J. Kelly, R.G. Wilson, J.M. Zavada, S.N.G. Chu, J.S. Lee and Z.G. Khim.

\section{References}

[1] http://www.almaden.ibm.com/sst/.

[2] H. Ohno, D. Chiba, F. Matsukura, T. Omiya, E. Abe, T. Dietl, Y. Ohno, K. Ohtani, Nature 408 (2000) 944.

[3] A. Oiwa, Y. Mitsumori, R. Moriya, T. Slupinski, H. Munekata, Phys. Rev. Lett. 88 (2002) 137202.

[4] Y.D. Park, A.T. Hanbicki, S.C. Erwin, C.S. Hellberg, J.M. Sullivan, J.E. Mattson, A. Wilson, G. Spanos, B.T. Jonker, Science 295 (2002) 651.

[5] H. Ohno, J. Vac. Sci. Technol. B 18 (2000) 2039.

[6] S.A. Wolf, D.D. Awschalom, R.A. Buhrman, J.M. Daughton, S. von Molnar, M.L. Roukes, A.Y. Chtchelkanova, D.M. Treger, Science 294 (2001) 1488.

[7] S. Von Molnar, et al., "World Technology (WTEC) Study on Spin Electronics: Highlights of Recent US Research and Development Activities", 2001, http://www.wtec.org/spin_US_summary.pdf.

[8] S.A. Chambers, Materials Today, April 2002, pp. 34-39.

[9] S. Das Sarma, Am. Scientist 89 (2001) 516. 
[10] H. Ohno, F. Matsukura, Y. Ohno, JSAP Int. 5 (2002) 4.

[11] D.D. Awschalom, J.M. Kikkawa, Science 287 (2000) 473.

[12] C. Gould, G. Schmidt, G. Richler, R. Fiederling, P. Grabs, L.W. Molenkamp, Appl. Surf. Sci. 190 (2002) 395.

[13] P.R. Hammar, B.R. Bennett, M.J. Yang, M. Johnson, Phys. Rev. Lett. 83 (1999) 203.

[14] F.G. Monzon, H.X. Tang, M.L. Roukes, Phys. Rev. Lett. 84 (2000) 5022.

[15] G.A. Prinz, "Magnetic metal films on semiconductor substrates", in: B. Heinrich, J.A.C. Bland (Eds.), Ultrathin Magnetic Structures II, Springer-Verlag, New York, 1994.

[16] G. Schmidt, D. Ferrand, L.W. Molenkamp, A.T. Filip, B.J. van Wees, Phys. Rev. B 62 (2000) R4793.

[17] E.I. Rashba, Phys. Rev. B 62 (2000) R16267.

[18] H.J. Zhu, M. Ramsteiner, H. Kostial, M. Wassermeier, H.-P. Schönherr, K.H. Ploog, Phys. Rev. Lett. 87 (2001) 016601.

[19] F. Matsukura, H. Ohno, A. Shen, Y. Sugawara, Phys. Rev. B 57 (1998) R2037.

[20] H. Ohno, A. Shen, F. Matsukura, A. Oiwa, A. Endo, S. Katsumoto, Y. Iye, Appl. Phys. Lett. 69 (1996) 363.

[21] R. Shioda, K. Ando, T. Hayashi, M. Tanaka, Phys. Rev. B 58 (1998) 1100.

[22] Y. Satoh, N. Inoue, Y. Nishikawa, J. Yoshino, in: H. Ohno, J. Yoshino, Y. Oka (Eds.), Proceedings of the Third Symposium on Physics and Applications of Spin-Related Phenomena in Semiconductors, Sendai, Japan, November 1997, p. 23.

[23] T. Hayashi, M. Tanaka, T. Nishinaga, H. Shimoda, H. Tsuchiya, Y. Otsuka, J. Cryst. Growth 175 (1997) 1063.

[24] A. Van Esch, L. Van Bockstal, J. de Boeck, G. Verbanck, A.S. vas Steenbergen, R.J. Wellman, G. Grietens, R. Bogaerts, F. Herlach, G. Borghs, Phys. Rev. B 56 (1997) 13103.

[25] B. Beschoten, P.A. Crowell, I. Malajovich, D.D. Awschalom, F. Matsukura, A. Shen, H. Ohno, Phys. Rev. Lett. 83 (1999) 3073.

[26] M. Tanaka, J. Vac. Sci. Technol. B 16 (1998) 2267.

[27] Y. Nagai, T. Kurimoto, K. Nagasaka, H. Nojiri, M. Motokawa, F. Matsukura, T. Dietl, H. Ohno, Jpn. J. Appl. Phys. 40 (2001) 6231.

[28] J. Sadowski, R. Mathieu, P. Svedlindh, J.Z. Domagala, J. Bak-Misiuk, J. Swiatek, M. Karlsteen, J. Kanski, L. Ilver, H. Asklund, V. Sodervall, Appl. Phys. Lett. 78 (2001) 3271.

[29] A. Shen, F. Matsukura, S.P. Guo, Y. Sugawara, H. Ohno, M. Tani, A. Abe, H.C. Liu, J. Cryst. Growth 201-202 (1999) 379.

[30] H. Shimizu, T. Hayashi, T. Nishinaga, M. Tanaka, Appl. Phys. Lett. 74 (1999) 398.

[31] B. Grandidier, J.P. Hys, C. Delerue, D. Stievenard, Y. Higo, M. Tanaka, Appl. Phys. Lett. 77 (2000) 4001.

[32] R.K. Kawakami, E. Johnson-Halperin, L.F. Chen, M. Hanson, N. Guebels, J.S. Speck, A.C. Gossard, D.D. Awschalom, Appl. Phys. Lett. 77 (2000) 2379.

[33] K. Ando, T. Hayashi, M. Tanaka, A. Twardowski, J. Appl. Phys. 83 (1998) 65481.

[34] D. Chiba, N. Akiba, F. Matsukura, Y. Ohno, H. Ohno, Appl. Phys. Lett. 77 (2000) 1873.

[35] H. Ohno, F. Matsukura, T. Owiya, N. Akiba, J. Appl. Phys. 85 (1999) 4277.

[36] T. Hayashi, M. Tanaka, T. Nishinaga, H. Shimada, J. Appl. Phys. 81 (1997) 4865.

[37] T. Hayashi, M. Tanaka, K. Seto, T. Nishinaga, K. Ando, Appl. Phys. Lett. 71 (1997) 1825.

[38] A. Twardowski, Mater. Sci. Eng. B 63 (1999) 96.

[39] T. Hayashi, M. Tanaka, A. Asamitsu, J. Appl. Phys. 87 (2000) 4673.

[40] N. Akiba, D. Chiba, K. Natata, F. Matsukura, Y. Ohno, H. Ohno, J. App. Phys. 87 (2000) 6436.

[41] S.J. Potashnik, K.C. Ku, S.H. Chun, J.J. Berry, N. Samarth, P. Schiffer, Appl. Phys. Lett. 79 (2001) 1495.

[42] G.M. Schott, W. Faschinger, L.W. Molenkamp, Appl. Phys. Lett. 79 (2001) 1807.

[43] H. Munekata, H. Ohno, S. von Molnar, A. Segmuller, L.L. Chang, L. Esaki, Phys. Rev. Lett. 63 (1989) 1849.

[44] K.J. Akai, Phys. Rev. Lett. 81 (1998) 3002.

[45] H. Ohno, H. Munekata, T. Penney, S. von Molnar, L. L Chang, Phys. Rev. Lett. 68 (1992) 2864.

[46] H. Munekata, A. Zaslevsky, P. Fumagalli, R.J. Gambino, Appl. Phys. Lett. 63 (1993) 2929.

[47] S. Koshihara, A. Oiwa, M. Hirasawa, S. Katsumoto, Y. Iye, C. Urano, H. Takagi, H. Munekata, Phys. Rev. Lett. 78 (1997) 4617.

[48] Y.L. Soo, S.W. Huang, Z.H. Ming, Y.H. Kao, H. Munekata, Phys. Rev. B 53 (1996) 4905.

[49] A. Oiwa, T. Slupinski, H. Munekata, Appl. Phys. Lett. 78 (2001) 518.

[50] Y. Nishikawa, A. Tackeuchi, M. Yamaguchi, S. Muto, O. Wada, IEEE J. Sel. Top. Quant. Electron. 2 (1996) 661.

[51] H. Munekata, in: Proceedings of the Papers Presented at ICCG-13, August 2001.

[52] I. Malajovich, J.M. Kikkawa, D.D. Awschalom, J.J. Berry, N. Samarth, Phys. Rev. Lett. 84 (2000) 1015.

[53] P.R. Hammar, B.R. Bennet, M.Y. Yang, M. Johnson, J. Appl. Phys. 87 (2000) 4665.

[54] A. Hirohata, Y.B. Xu, C.M. Guetler, J.A.C. Bland, J. Appl. Phys. 87 (2000) 4670.

[55] M. Johnson, J. Vac. Sci. Technol. A 16 (1998) 1806.

[56] S. Cardelis, C.G. Smith, C.H.W. Barnes, E.H. Linfield, J. Ritchie, Phys. Rev. B 60 (1999) 7764.

[57] R. Fiederling, M. Kein, G. Rerescher, W. Ossan, G. Schmidt, A. Wang, L.W. Molenkamp, Nature 402 (1999) 787.

[58] G. Borghs, J. De Boeck, Mater. Sci. Eng. B 84 (2001) 75.

[59] Y. Ohno, D.K. Young, B. Bescholen, F. Matsukura, H. Ohno, D.D. Awschalom, Nature 402 (1999) 790. 
[60] B.T. Jonker, Y.D. Park, B.R. Bennett, H.D. Cheong, G. Kioseoglou, A. Petrou, Phys. Rev. B 62 (2000) 8180.

[61] Y.D. Park, B.T. Jonker, B.R. Bennett, G. Itskos, M. Furis, G. Kioseoglou, A. Petrou, App. Phys. Lett. 77 (2000) 3989.

[62] G. Schmidt, D. Ferrand, L.W. Molenkamp, A.T. Filip, B.J. van Wees, Phys. Rev. B 62 (2000) R4790.

[63] Y.Q. Jin, R.C. Shi, S.J. Chou, IEEE Trans. Magn. 32 (1996) 4707.

[64] C.M. Hu, J. Nitta, A. Jensen, J.B. Hansen, H. Takayanagai, Phys. Rev. B 63 (2001) 125333.

[65] F.G. Monzon, H.X. Tang, M.L. Roukes, Phys. Rev. Lett. 84 (2000) 5022.

[66] S. Gardelis, C.G. Smith, C.H.W. Barnes, F. Matsukura, H. Ohno, Jpn. J. Appl. Phys. 40 (2001) L1274.

[67] H. Breve, S. Nemeth, Z. Liu, J. De Boeck, G. Borghs, J. Magn. Magn. Mater. 226-230 (2001) 933.

[68] H. Munekata, H. Ohno, S. von Molnar, A. Segmuller, L.L. Chang, L. Esaki, Phys. Rev. Lett. 63 (1989) 1849.

[69] H.J. Zhu, M. Ramsteiner, H. Kostial, M. Wassermeier, H.P. Schononherr, K.H. Ploog, Phys. Rev. Lett. 87 (2001) 016601.

[70] M. Kohda, Y. Ohno, K. Takamura, F. Matsukura, H. Ohno, Jpn. J. Appl. Phys. 40 (2001) L1274.

[71] J.A. Katine, F.J. Albert, R.A. Buhrman, E.D. Myers, D.C. Ralph, Phys. Rev. Lett. 84 (2000) 319.

[72] J. Nitta, T. Ahazaki, H. Takayanngi, T. Enoki, Phys. Rev. Lett. 78 (1997) 1335.

[73] S. Cho, S. Choi, G.B. Cha, S.C. Hong, Y. Kim, Y.-J. Zhao, A.J. Freeman, J.B. Ketterson, B.J. Kim, Y.C. Kim, B.C. Choi, Phys. Rev. Lett. 88 (2002) 257203-1.

[74] G.A. Medvedkin, T. Ishibashi, T. Nishi, K. Hiyata, Jpn. J. Appl. Phys. 39 (2000) L949.

[75] G.A. Medvedkin, K. Hirose, T. Ishibashi, T. Nishi, V.G. Voevodin, K. Sato, J. Cryst. Growth 236 (2002) 609.

[76] S. Choi, G.B. Cha, S.C. Hong, S. Cho, Y. Kim, J.B. Ketterson, S.-Y. Jeong, G.C. Yi, Solid-State Commun. 122 (2002) 165.

[77] K. Ueda, H. Tahata, T. Kawai, Appl. Phys. Lett. 79 (2001) 988.

[78] Y. Matsumoto, M. Murakami, T. Shono, H. Hasegawa, T. Fukumura, M. Kawasaki, P. Ahmet, T. Chikyow, S. Koshikara, H. Koinuma, Science 291 (2001) 854.

[79] F. Holtzberg, S. von Molnar, J.M.D. Coey, in: T. Moss (Ed.), Handbook on Semiconductors, North-Holland, Amsterdam, 1980.

[80] T. Dietl, H. Ohno, F. Matsukura, J. Cibert, D. Ferrand, Science 287 (2000) 1019.

[81] C. Zener, Phys. Rev. B 81 (1951) 440.

[82] M. Van Schilfgaarde, O.N. Myrasov, Phys. Rev. B 63 (2001) 233205.

[83] T. Dietl, H. Ohno, F. Matsukura, Phys. Rev. B 63 (2001) 195205.

[84] T. Dietl, J. Appl. Phys. 89 (2001) 7437.

[85] T. Jungwirth, W.A. Atkinson, B. Lee, A.H. MacDonald, Phys. Rev. B 59 (1999) 9818.

[86] M. Berciu, R.N. Bhatt, Phys. Rev. Lett. 87 (2001) 108203.

[87] R.N. Bhatt, M. Berciu, M.D. Kennett, X. Wan, J. Supercond.: Incorp. Novel Magn. 15 (2002) 71.

[88] V.I. Litvinov, V.A. Dugaev, Phys. Rev. Lett. 86 (2001) 5593.

[89] J. Konig, H.H. Lin, A.H. MacDonald, Phys. Rev. Lett. 84 (2001) 5628.

[90] J. Schliemann, J. Konig, A.H. MacDonald, Phys. Rev. B 64 (2001) 165201.

[91] T. Jungwirth, J. Konig, J. Sinova, J. Kucera, A.H. MacDonald, Phys. Rev. B 89 (2002) 107303.

[92] C.R. Abernathy, Mater. Sci. Rep. R16 (1995) 203.

[93] R.Y. Kororkov, J.M. Gregie, B.W. Wessels, Appl. Phys. Lett. 80 (2002) 1731.

[94] H. Katayama-Yoshida, R. Kato, T. Yamamoto, J. Cryst. Growth 231 (2001) 438.

[95] I.D. Goepfert, E.F. Schubert, A. Osinsky, P.E. Norris, N.N. Faleev, J. Appl. Phys. 88 (2000) 2030

[96] K. Sato, H. Katayama-Yoshida, Jpn. J. Appl. Phys. 40 (2001) L485.

[97] N. Theodoropoulou, A.F. Hebard, M.E. Overberg, C.R. Abernathy, S.J. Pearton, S.N.G. Chu, R.G. Wilson, Phys. Rev. Lett. 89 (2002) 107203-1.

[98] M.E. Overberg, B.P. Gila, G.T. Thaler, C.R. Abernathy, S.J. Pearton, N. Theodoropoulou, K.T. McCarthy, S.B. Arnason, A.F. Hebard, S.N.G. Chu, R.G. Wilson, J.M. Zavada, Y.D. Park, J. Vac. Sci. Technol. B 20 (2002) 969.

[99] M. Zajac, J. Gosk, M. Kaminska, A. Twardowski, T. Szyszko, S. Podliasko, Appl. Phys. Lett. 79 (2001) 2432.

[100] M.L. Reed, M.K. Ritums, H.H. Stadelmaier, M.J. Reed, C.A. Parker, S.M. Bedair, N.A. El-Masry, Mater. Lett. 51 (2001) 500.

[101] M.L. Reed, N.A. El-Masry, H. Stadelmaier, M.E. Ritums, N.J. Reed, C.A. Parker, J.C. Roberts, S.M. Bedair, Appl. Phys. Lett. 79 (2001) 3473.

[102] N. Theodoropoulou, A.F. Hebard, M.E. Overberg, C.R. Abernathy, S.J. Pearton, S.N.G. Chu, R.G. Wilson, Appl. Phys. Lett. 78 (2001) 3475.

[103] S. Sonoda, S. Shimizu, T. Sasaki, Y. Yamamoto, H. Hori, J. Cryst. Growth 237-239 (2002) 1358.

[104] T. Sasaki, S. Sonoda, Y. Yamamoto, K. Suga, S. Shimizu, K. Kindo, H. Hori, J. Appl. Phys. 91 (2002) 7911.

[105] G.T. Thaler, M.E. Overberg, B. Gila, R. Frazier, C.R. Abernathy, S.J. Pearton, J.S. Lee, S.Y. Lee, Y.D. Park, Z.G. Khim, J. Kim, F. Ren, Appl. Phys. Lett. 80 (2002) 3964.

[106] S.E. Park, H.-J. Lee, Y.C. Cho, S.-Y. Jeong, C.R. Cho, S. Cho, Appl. Phys. Lett. 80 (2002) 4187.

[107] M. Hashimoto, Y.-K. Zhou, M. Kanamura, H. Asahi, Solid-State Commun. 122 (2002) 37.

[108] M. Yamada, J. Appl. Phys. 91 (2002) 7908. 
[109] K. Inumaru, H. Okamoto, S.J. Yamanaka, J. Cryst. Growth 239-279 (2002) 2050.

[110] K. Suzuki, T. Kaneko, H. Yoshida, H. Morita, H. Fujimori, J. Alloys Compd. 224 (1995) 232.

[111] Y.L. Soo, G. Kioseoglou, S. Kim, S. Huang, Y.H. Kaa, S. Kubarawa, S. Owa, T. Kondo, H. Munekata, Appl. Phys. Lett. 79 (2001) 3926.

[112] M. Sato, H. Tanida, K. Kato, T. Sasaki, Y. Yamamoto, S. Sonoda, S. Shimiyu, H. Hori, J. Jpn. Appl. Phys. 41 (2002) 4513.

[113] N.A. Theodoropoulou, A.F. Hebard, S.N.G. Chu, M.E. Overberg, C.R. Abernathy, S.J. Pearton, R.G. Wilson, J.M. Zavada, Appl. Phys. Lett. 79 (2001) 3452.

[114] S.J. Pearton, M.E. Overberg, G. Thaler, C.R. Abernathy, N. Theodoropoulou, A.F. Hebard, S.N.G. Chu, R.G. Wilson, J.M. Zavada, A.Y. Polyakov, A. Osinsky, Y.D. Park, J. Vac. Sci. Technol. A 20 (2002) 583.

[115] H. Akinaga, S. Nemeth, J. De Boeck, L. Nistor, H. Bender, G. Borghs, H. Ofuchi, M. Oshima, Appl. Phys. Lett. 77 (2000) 4377.

[116] M. Hashimoto, Y.Z. Zhou, M. Kanamura, H. Asahi, Solid-State Commun. 122 (2002) 37.

[117] K. Sato, H. Katayama-Yoshida, Jpn. J. Appl. Phys. 40 (2001) L334.

[118] K. Sato, H. Katayama-Yoshida, Jpn. J. Appl. Phys. 39 (2000) L555.

[119] K. Sato, H. Katayama-Yoshida, Physica E 10 (2001) 251.

[120] K. Sato, H. Katayama-Yoshida, Mater. Res. Soc. Symp. Proc. 666 (2001) F4.6.1.

[121] T. Yamamoto, H. Katayama-Yoshida, Jpn. J. Appl. Phys. 38 (1999) L166.

[122] T. Fukumura, Z. Jin, M. Kawasaki, T. Shono, T. Hasegawa, S. Koshikara, S. Koshihara, H. Koinuma, Appl. Phys. Lett. 78 (2001) 958.

[123] T. Wakano, N. Fujimura, Y. Morinaga, N. Abe, A. Ashida, T. Ito, Physica E 10 (2001) 260.

[124] Z. Jin, K. Hasegawa, T. Fukumura, Y.Z. Yoo, T. Hasegawa, H. Koinuma, M. Kawasaki, Physica E 10 (2001) 256.

[125] S.W. Jung, S.-J. An, G.-C. Yi, C.U. Jung, S.-I. Lee, S. Cho, Appl. Phys. Lett. 80 (2002) 4561.

[126] K. Ueda, H. Tabota, T. Kamai, Appl. Phys. Lett. 79 (2001) 988.

[127] S.A. Chambers, S. Thevuthascan, R.F.C. Farrow, R.G. Marks, J.U. Thiele, L. Folks, M.G. Sammant, A.T. Kellock, N. Ruzycki, D.L. Ederer, U. Diebold, Appl. Phys. Lett. 79 (2001) 3467.

[128] Y. Matsumoto, M. Murakami, T. Shono, T. Hasegawa, T. Fukumura, M. Kawasaki, P. Ahmet, T. Chikyow, S. Koshikara, H. Koinuma, Science 291 (2001) 854.

[129] P.A. Cox, Transition Metal Oxides, Oxford University Press, New York, 1992.

[130] D.S. Ginley, C. Bright (Eds.) MRS Bulletin, vol. 25, no. 8, August 2000.

[131] H.L. Hartnagel, A.L. Dawar, A.K. Jain, C. Jagadish, "Semiconducting Transparent Thin-Films, IOP Publishing, London, 1995.

[132] D.H. Kim, J.S. Yang, K.W. Lee, S.D. Bu, T.W. Noh, S.J. Oh, Y. Kim, J. Chang, H. Tananka, H. Lee, T. Kawai, Appl. Phys. Lett. 81 (2002) 2421

[133] S.J. Pearton, M.E. Overberg, C.R. Abernathy, N.A. Theodoropoulou, A.F. Hebard, S.N.G. Chu, A. Osinsky, V. Zuflyigin, L.D. Zhu, A.Y. Polyakov, R.G. Wilson, J. Appl. Phys. 92 (2002) 2047.

[134] R. Fiederling, M. Kein, G. Resescher, W. Ossau, G. Schmidt, W. Wang, L.W. Molenkamp, Nature 402 (1999) 787.

[135] H. Fukumura, Z. Jin, A. Ohtomo, H. Koinuma, M. Kawasaki, Appl. Phys. Lett. 75 (1999) 3366.

[136] H. Tang, H. Berger, P.E. Schmid, F. Levy, Solid-State Commun. 92 (1994) 267.

[137] M.E. Tobar, J. Krupka, E.N. Ivanov, R.A. Woode, J. Appl. Phys. 83 (1998) 1604.

[138] H. Tang, K. Prasad, R. Sanjines, P.E. Schmid, F. Levy, J. Appl. Phys. 75 (1994) 2042.

[139] E. Yagi, R.R. Hasiguti, M. Aono, Phys. Rev. B 54 (1996) 7945.

[140] J.W. DeFord, O.W. Johnson, J. Appl. Phys. 54 (1983) 889.

[141] Y. Ohno, D.K. Young, B. Beschoten, F. Matsukura, H. Ohno, D.D. Awschalom, Nature 402 (1999) 790.

[142] B.T. Jonker, Y.D. Park, B.R. Bennet, H.D. Cheong, G. Kioseoglou, A. Petrou, Phys. Rev. B 62 (2000) 8180.

[143] Y.D. Park, B.T. Jonker, B.R. Bennet, G. Itzkos, M. Furis, G. Kioseoglou, A. Petrou, Appl. Phys. Lett. 77 (2000) 3989.

[144] B.T. Jonker, A.T. Hanbicki, Y.D. Park, G. Itskos, M. Furis, G. Kioseoglou, A. Petrou, Appl. Phys. Lett. 79 (2001) 3098.

[145] P. LeClair, J.K. Ha, H.J.M. Swagten, J.T. Kohlhepp, C.H. van de Vin, W.J.M. de Jonge, Appl. Phys. Lett. 80 (2002) 625.

[146] A. Kaminski, S. Das Sarma, Phys. Rev. Lett. 88 (2002) 247202-1.

[147] L. Kronik, M. Jain, J.R. Chelikowsky, Phys. Rev. B 66 (2002) 041203.

[148] E. Kulakov, H. Nakagawa, H. Mariette, H. Ohta, Y.A. Uspenski, Phys. Rev. B 66 (2002) 045203.

[149] M.P. Kennett, M. Berciu, R.N. Bhatt, Phys. Rev. B 66 (2002) 045207.

[150] T. Dietl, F. Matsukura, H. Ohno, Phys. Rev. B 66 (2002) 033201.

[151] A.C. Durst, R.N. Bhatt, P.A. Wolf, Phys. Rev. B 65 (2002) 235205.

[152] G. Bouzerar, T.P. Pareek, Phys. Rev. B 65 (2002) 153203.

[153] A.L. Chudnoviskiy, D. Pfannkoche, Phys. Rev. B 65 (2002) 165216.

[154] N. Teraguchi, A. Suzuki, Y. Nannishi, Y.K. Zhou, M. Hashimoto, H. Asashi, Solid-State Commun. 122 (2002) 651.

[155] S.G. Yang, A. Pakhomov, S. Hung, C. Wong, Appl. Phys. Lett. 81 (2002) 2148. 